\title{
Old open clusters as key tracers of Galactic chemical evolution
}

\section{Iron and elemental abundances in NGC 2324, NGC 2477, NGC 2660, NGC 3960, and Berkeley $32^{\star, \star \star}$}

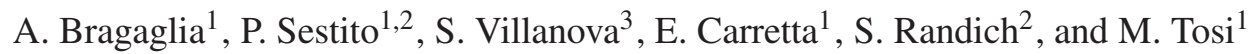 \\ 1 INAF - Osservatorio Astronomico di Bologna, via Ranzani 1, 40127 Bologna, Italy \\ e-mail: [angela.bragaglia; eugenio.carretta;monica.tosi]@oabo.inaf.it \\ 2 INAF - Osservatorio Astronomico di Arcetri, Largo E. Fermi 5, 50125 Firenze, Italy \\ e-mail: [randich;sestito] @arcetri.astro.it \\ 3 Dipartimento di Astronomia, Università di Padova, Vicolo Osservatorio 2, 35122 Padova, Italy \\ e-mail: sandro.villanova@unipd.it
}

Received 16 May 2007 / Accepted 27 November 2007

\begin{abstract}
Aims. To constrain the formation and chemical evolution of the Galactic disk, we surveyed open clusters of different ages, metal contents, and distances from the Galactic centre. We employed FLAMES on VLT-UT2 to collect UVES spectra of five to ten giant stars in each of the selected clusters and used them to derive the iron abundance and the detailed chemical composition. Methods. Equivalent widths were measured and abundance analysis was performed using the MOOG code and Kurucz model atmospheres on all stars considered as cluster members on the basis of their radial velocity.

Results. We derived the atmospheric parameters and the Fe abundance for NGC 2324 and NGC 2477 (average $[\mathrm{Fe} / \mathrm{H}]=-0.17$ with rms 0.05 dex, and +0.07 with rms 0.03 dex, respectively), two clusters never analyzed using high resolution spectra. We also derived the abundances of $\mathrm{Mg}, \mathrm{Al}, \mathrm{Ca}, \mathrm{Si}, \mathrm{Ti}, \mathrm{Cr}, \mathrm{Ni}$, and $\mathrm{Ba}$ for these two clusters and for NGC 2660, NGC 3960, and Berkeley 32, whose atmospheric parameters and metallicities were measured in a previous paper. We determined the reddening values for the five clusters, based on the spectroscopic temperatures, literature photometry, and a colour-temperature relation.

Conclusions. All clusters show solar-scaled abundances for $\alpha$ - and Fe-peak elements, while $[\mathrm{Na} / \mathrm{Fe}]$ appears slightly enhanced and $[\mathrm{Ba} / \mathrm{Fe}]$ significantly enhanced. Our findings were compared to thin-disk stars and other open clusters, and no significant deviation from the standard behavior was found.
\end{abstract}

Key words. stars: evolution - stars: abundances - Galaxy: disk - open clusters and associations: general

\section{Introduction}

Open clusters (OCs) are very good tracers of the Galactic disk properties, of its formation and evolution (e.g., Panagia \& Tosi 1981; Friel 1995; Twarog et al. 1997; Freeman \& Bland-Hawthorn 2002). In particular, OCs may be used to study the metallicity distribution in the disk and its possible variation with time; this has important bearings on the formation mechanism and on the chemical evolution. Several papers have been devoted to the derivation of the metallicity of OCs covering a large interval of ages and galactic positions, to study the abundance distribution with Galactocentric distance (see e.g., the discussions in Twarog et al. 1997; Friel et al. 2002; Yong et al. 2005; Friel 2006; Bragaglia \& Tosi 2006; and Sestito et al. 2006, hereafter Paper I).

One of the requirements for such a study is a large sample of OCs with all properties homogeneously determined (i.e., all on the same scale). We are working in this direction and observed nine OCs covering a large range in age (from about 0.5

\footnotetext{
* Based on observations collected at ESO telescopes under programmes 072.D-0550 and 074.D-0571.

$\star \star$ Tables 4, 6-10, 13, and 14 are only available in electronic form at the CDS via anonymous ftp to cdsarc.u-strasbg.fr (130.79.128.5) or via http://cdsweb.u-strasbg.fr/cgi-bin/qcat?]/A+A/ $480 / 79$
}

to $6 \mathrm{Gyr})$, in metallicity ([Fe/H] from about -0.5 to +0.5$)$ and in Galactocentric distances (from about 7 to $21 \mathrm{kpc}$ ) to derive their chemical composition.

As described in Randich et al. (2005) we collected spectra of stars in several OCs using FLAMES/GIRAFFE and FLAMES/UVES (Pasquini et al. 2002) mounted on VLT-UT2. The GIRAFFE spectra were obtained to derive cluster radial velocities and membership and to study the Li abundance in main sequence stars of clusters of different age and metallicity, and the UVES spectra to measure the detailed chemical composition. In Paper I we presented our project and derived the atmospheric parameters and the metallicities of the stars observed with FLAMES/UVES in the first three OCs of our sample: NGC 2660, NGC 3960, and Berkeley 32. In Sestito et al. (2007, hereafter S07) we concentrated on NGC 6253, the most metal rich cluster of our sample. Here, we present the metallicity and detailed elemental abundances of two more OCs: NGC 2324 and NGC 2477. Neither cluster has previously been the subject of an abundance analysis based on high resolution spectra. We also present the detailed chemical abundances of light, $\alpha$ - and Fe-group elements for the three clusters of Paper I; this is the first analysis of the kind for NGC 2660 and Be 32, while three stars in NGC 3960 have already been studied by Bragaglia et al. (2006, hereafter B06). 
Table 1. Observation log for NGC 2324 and NGC 2477.

\begin{tabular}{lcccc}
\hline \hline Date & UT $_{\text {beginning }}$ & Exptime & Grating & Stars \\
\hline \multicolumn{5}{c}{ NGC 2324 } \\
2005-01-20 & $05: 35: 11$ & 3600 & CD3 & 8 \\
$2005-01-20$ & $06: 36: 12$ & 3900 & CD3 & 8 \\
$2005-01-23$ & $05: 00: 48$ & 3600 & CD4 & 8 \\
$2005-01-23$ & $06: 01: 54$ & 3600 & CD4 & 8 \\
\multicolumn{5}{c}{ NGC 2477 } \\
$2004-04-19$ & $00: 39: 02$ & 2595 & CD4 & 6 \\
$2004-04-19$ & $01: 34: 18$ & 2595 & CD3 & 6 \\
$2004-05-08$ & $23: 41: 52$ & 2595 & CD3 & 5 \\
$2004-05-09$ & $00: 36: 39$ & 2595 & CD4 & 5 \\
\hline
\end{tabular}

We recall that NGC 2660 and NGC 3960 have an age of about 1 Gyr (Sandrelli et al. 1999; Prisinzano et al. 2004; B06) and nearly solar metallicity, while Be 32 is about 6 Gyr old (Kaluzny \& Mazur 1991) or slightly less (Tosi et al. 2007: about $5.3 \mathrm{Gyr})$ and has $[\mathrm{Fe} / \mathrm{H}]$ about -0.3 dex. All metallicities are taken from Paper I, where a detailed summary of previous studies on these OC is reported.

Information on the FLAMES pontings of the two new clusters are given in Table 1, where the exposure times are in seconds. In NGC 2324 the same eight stars were targeted in all pointings, so they were observed for two hours in each grating. In NGC 2477 the two main sequence stars were observed in the two pointings with the same grating, so we have a total of nine stars. CD3 and CD4 cover the 4800-6800 and 6700-10000 $\AA$ range, respectively, and the resolution is $R \simeq$ 45000 . In Table 2 we list the main properties of the observed stars; identifications and coordinates are taken from the EIS catalogues ${ }^{1}$. The $B V I$ magnitudes come from Kyeong et al. or Piatti et al. (NGC 2324) and the EIS catalogue corrected to the Kassis et al. photometry (NGC 2477), while $J K$ come from 2MASS. The $S / N$ ratios were computed in two regions, near 5600 and $6300 \AA$. The radial velocities $(R V \mathrm{~s})$, in $\mathrm{km} \mathrm{s}^{-1}$, are heliocentric and have an error of about $0.5 \mathrm{~km} \mathrm{~s}^{-1}$. In the last column, rot. indicates a star with very large lines, likely to be a fast rotator, SB a binary star, MS a main sequence stars, and NM? a possible non member; a "*" symbol indicates that the $R V$ is present also in Mermilliod et al. (2001) or Eigenbrod et al. (2004) for NGC 2324 and NGC 2477, respectively.

Both NGC 2324 and NGC 2477 have an age of $\sim 1$ Gyr or slightly lower. The former OC was recently investigated by Piatti et al. (2004) who provided photometry in the JohnsonCousins $V I_{\mathrm{c}}$ filters and the Washington system $C$ and $T_{1}$ passbands; for NGC 2324 they derived $E(B-V)=0.25$, distance $3.8 \mathrm{kpc}$ and $[\mathrm{Fe} / \mathrm{H}]=-0.31$ from their best-fit isochrone. Kyeong et al. (2001) presented UBVIC CCD photometry finding $E(B-V)=0.17,(m-M)_{0}=13.1,[\mathrm{Fe} / \mathrm{H}] \sim-0.3$, and an age of 600-700 Myr from a comparison with isochrones. We used their photometry, obtained through the $\mathrm{WEBDA}^{2}$, to independently check the cluster properties and reached almost the same results (i.e., age of $0.6 \mathrm{Gyr},[\mathrm{Fe} / \mathrm{H}]=-0.30,(m-M)_{0}=13.1$, and $E(B-V)=0.18$, see Fig. 1). Mermilliod et al. (2001) obtained accurate radial velocities $(R V \mathrm{~s})$ and photoelectric $U B V$ photometry for 17 candidate giant stars in NGC 2324, measuring $\langle R V\rangle=+41.81 \pm 0.25 \mathrm{~km} \mathrm{~s}^{-1}$ and finding several long period binaries (see their Tables 3 and 2).

\footnotetext{
1 Alternative identifications come from Kyeong et al. (2001: ID $\left._{1}\right)$ and Piatti et al. (2004: $\mathrm{ID}_{2}$ ) for NGC 2324, from Kassis et al. (1997: ID 1 ) and the WEBDA $\left(\mathrm{ID}_{2}\right)$ for NGC 2477.

${ }^{2}$ http://www . univie.ac.at/webda/webda.html
}

More numerous investigations have been published for NGC 2477. Among the first comprehensive studies, Hartwick et al. (1972), on the basis of $U B V$ photographic and photoelectric observations and DDO data, derived $(m-M)_{0}=10.61 \pm$ 0.21 , differential reddening $[E(B-V)$ from about 0.2 to 0.4$]$, an age of $1.5 \mathrm{Gyr}$ and a metallicity about 1.5 times that of the Hyades. More recently, NGC 2477 was studied by Kassis et al. (1997), who used much deeper BVI CCD photometry to derive, on the basis of isochrone fitting (assuming $[\mathrm{Fe} / \mathrm{H}]=-0.05 \pm$ 0.11 from Friel \& Janes 1993 and reddening 0.2-0.4 from Hartwick et al.), an age of $1_{-0.2}^{+0.3} \mathrm{Gyr}$ and $(m-M)_{0}=10.5_{-0.3}^{+0.4}$. To derive the initial atmospheric parameters we adopted age $=$ $1 \mathrm{Gyr},[\mathrm{Fe} / \mathrm{H}]=-0.13,(m-M)_{0}=10.4$, and $E(B-V)=0.28$. Finally, Eigenbrod et al. (2004) presented accurate radial velocities and photoelectric $U B V$ photometry of red giant candidates in the field of this rich OC. From 49, constant velocity, member stars they derived an average radial velocity of $\langle R V\rangle=7.32 \pm$ $0.13 \mathrm{~km} \mathrm{~s}^{-1}$. Combining their results with the extensive photometry by Momany et al. (2001) and assuming the metallicities by Friel et al. (2002), they estimated a spread in reddening and an age of about 1 Gyr. They also confirmed a large cluster mass (at least $5300 M_{\odot}$ ).

The only (low-resolution) spectroscopic study intended to derive the metallicity of NGC 2324 and NGC 2477 is by Friel et al. (2002): for NGC 2324 they found $[\mathrm{Fe} / \mathrm{H}]=-0.15(\sigma=$ $0.16)$ on the basis of seven stars and adopting $E(B-V)=0.20$, while for NGC 2477 they assumed $E(B-V)=0.29$, and derived $[\mathrm{Fe} / \mathrm{H}]=-0.13(\sigma=0.10)$ on the basis of 28 stars.

The layout of the present paper is the following: we present the method of analysis in Sect. 2, the derived abundances and a discussion in Sect. 3; a summary is given in Sect. 4. Reddening values derived on the basis of our spectroscopic analysis are presented in the Appendix.

\section{Observations and abundance analysis}

The selection of the targets (almost all red clump giants), the observations, the reductions, the identification of member stars on the basis of radial velocities were already described in Paper I for NGC 2660, NGC 3960, and Be 32. We further add here that the spectra were corrected for telluric contamination near the $\mathrm{Na}$ I lines at 8183-8194 $\AA$. We used a rapidly rotating early type star observed with UVES for another programme and the IRAF ${ }^{3}$ task TELLURIC.

Table 1 lists the log of the observations. The parameters for NGC 2324 were chosen as described in the previous section; the age of NGC 2477 was taken from Kassis et al. (1997), the metallicity from Friel et al. (2002), the distance modulus and reddening from the WEBDA. Figure 1 shows the position on the colour-magnitude diagrams of the observed stars for NGC 2324 (together with the best-fit isochrone) and NGC 2477. Figure 2 shows small portions of the spectra of the eight and nine stars observed in NGC 2324 and NGC 2477, respectively.

Atmospheric parameters for the two new OCs were derived in the same way as for the three others. We determined input temperatures and gravities from the $B-V$ and $V-K$ colours, and the relations by Alonso et al. (1999). Given the ages of $\sim 1 \mathrm{Gyr}$ we adopted a mass of $\sim 2 M_{\odot}$ for both clusters. The final temperatures and gravities were obtained spectroscopically, using

\footnotetext{
3 IRAF is distributed by the National Optical Astronomical Observatory, which are operated by the Association of Universities for Research in Astronomy, under contract with the National Science Foundation.
} 

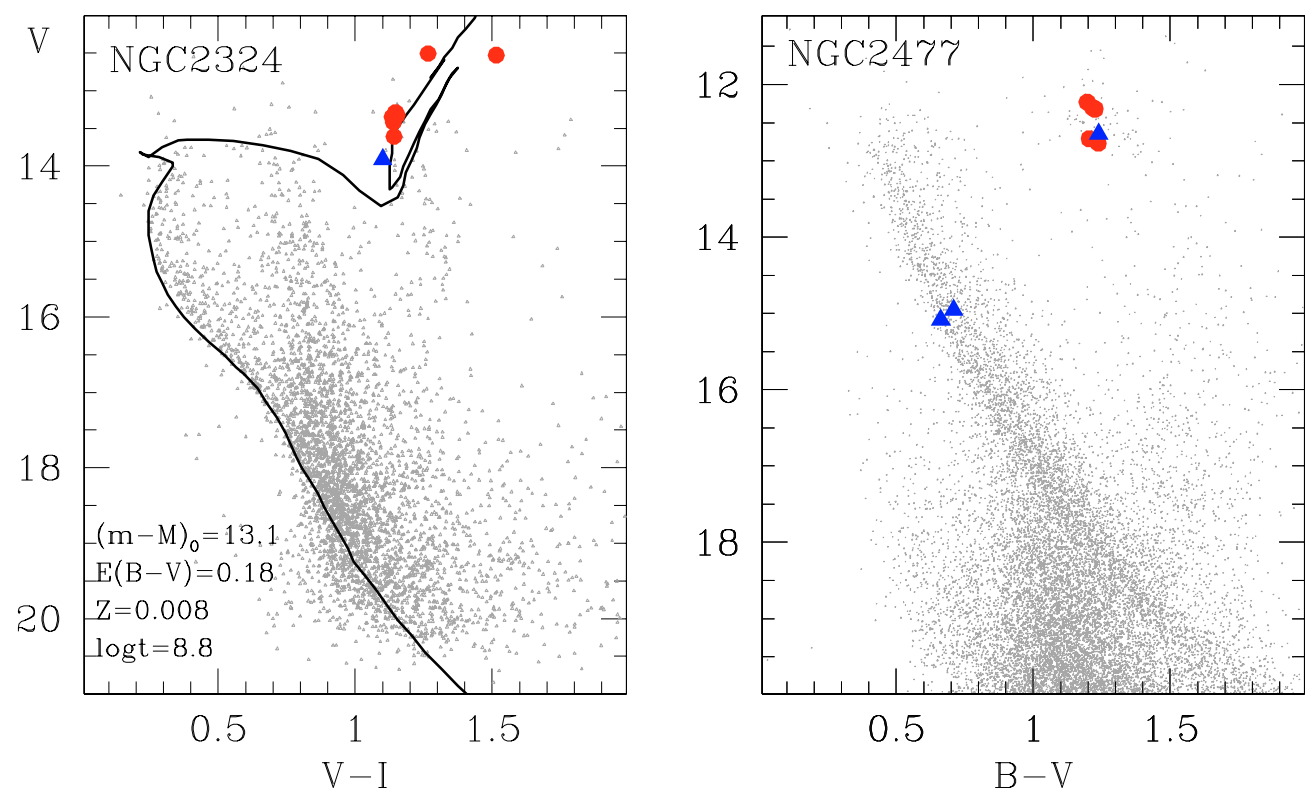

Fig. 1. Left panel: CMD for NGC 2324 (photometry from Kyeong et al. 2001); we also show the best-fit isochrone (from the Padova set by Bertelli et al. 1994) corresponding to the values given in the box and adopted in the paper. Right panel: CMD for NGC 2477 (photometry from EIS, aligned to the Kassis et al. 1997, one). In both panels the (red) filled circles indicate stars for which we derived abundances, and the (blue) triangles discarded stars (see text).

Table 2. Information on the stars observed in NGC 2324 and NGC 2477.

\begin{tabular}{|c|c|c|c|c|c|c|c|c|c|c|c|c|}
\hline ID & $\mathrm{ID}_{1}$ & $\mathrm{ID}_{2}$ & $\overline{\mathrm{RA}}$ & $\overline{\text { Dec }}$ & $\overline{\bar{B}}$ & $\overline{\bar{V}}$ & $\bar{I}$ & $\bar{J}$ & $\overline{\bar{K}}$ & $\overline{S S / N}$ & $\overline{\overline{R V}}$ & $\overline{\text { Notes }}$ \\
\hline \multicolumn{13}{|c|}{ NGC 2324} \\
\hline 010360 & 2633 & 788 & 07:04:22.511 & 01:06:16.68 & 14.863 & 13.915 & 12.814 & 12.059 & 11.439 & $80-85$ & 39.07 & rot. \\
\hline 010488 & 1439 & 850 & 07:04:01.151 & 01:06:04.09 & 14.347 & 13.351 & 12.216 & 11.335 & 10.723 & $100-105$ & 42.16 & * \\
\hline 010779 & & 2603 & 07:04:00.324 & 00:58:16.95 & & 12.530 & 11.015 & 9.945 & 9.046 & $150-180$ & 42.14 & * \\
\hline 010787 & 1607 & 2225 & 07:04:03.774 & 01:00:14.89 & 14.444 & 13.422 & 12.283 & 11.438 & 10.803 & $95-130$ & 45.85 & $\mathrm{SB}, *$ \\
\hline 010797 & 1867 & 1006 & 07:04:08.229 & 01:05:18.57 & 14.312 & 13.291 & 12.144 & 11.294 & 10.633 & $100-110$ & 34.91 & $\mathrm{SB}, *$ \\
\hline 010828 & & 1992 & 07:04:16.174 & 01:01:13.53 & & 12.508 & 11.242 & 10.284 & 9.571 & $130-180$ & 41.78 & * \\
\hline 030878 & 2179 & 1788 & 07:04:13.555 & 01:02:05.27 & 14.635 & 13.608 & 12.466 & 11.628 & 10.980 & $85-105$ & 41.82 & * \\
\hline 030957 & \multicolumn{11}{|c|}{ NGC 2477} & $\mathrm{SB}, *$ \\
\hline 006530 & 4954 & 7053 & $07: 51: 58.755$ & $-38: 31: 43.11$ & 15.747 & 15.084 & 14.232 & 13.527 & 13.207 & $40-45$ & 6.97 & MS \\
\hline 007109 & 1566 & 2078 & $07: 52: 35.475$ & $-38: 30: 20.87$ & 15.668 & 14.958 & 14.055 & 13.324 & 13.006 & $35-40$ & 10.74 & MS \\
\hline 036280 & 2447 & 13385 & $07: 52: 25.298$ & $-38: 38: 38.70$ & 14.008 & 12.771 & & 10.311 & 9.631 & $95-130$ & 7.02 & \\
\hline 036284 & 694 & 10960 & $07: 52: 45.855$ & $-38: 38: 10.82$ & 13.889 & 12.650 & 11.369 & 10.360 & 9.692 & $100-140$ & 3.97 & NM? \\
\hline 036288 & 1492 & 4221 & $07: 52: 36.507$ & $-38: 37: 54.35$ & 13.428 & 12.231 & 10.995 & 10.037 & 9.363 & $115-150$ & 8.27 & * \\
\hline 036326 & 3838 & 5035 & 07:52:10.735 & $-38: 35: 16.26$ & 13.523 & 12.306 & & 10.023 & 9.376 & $135-190$ & 6.71 & $\mathrm{SB}, *$ \\
\hline 036363 & & 3206 & 07:52:55.011 & $-38: 33: 12.62$ & 13.544 & 12.321 & & 10.024 & 9.384 & $130-190$ & 6.80 & * \\
\hline 036385 & 1019 & 2061 & $07: 52: 41.812$ & $-38: 32: 13.00$ & 13.914 & 12.710 & & 10.382 & 9.747 & $100-135$ & 8.06 & $\mathrm{SB}, *$ \\
\hline 036449 & 4763 & 8039 & 07:52:00.773 & $-38: 28: 49.09$ & 13.546 & 12.320 & 11.024 & 10.034 & 9.344 & $125-165$ & 8.18 & \\
\hline
\end{tabular}

the excitation and ionization equilibria of iron. Microturbulent velocities were derived as in Paper I using the relation with surface gravity derived by Carretta et al. (2004b): $\xi=[1.5-0.13 \times$ $\log g] \mathrm{km} \mathrm{s}^{-1}$.

Chemical abundance analysis was carried out using the updated version of the package MOOG ${ }^{4}$ (Sneden 1973) and adopting the model atmospheres by Kurucz (1993).

Our final sample contains five stars in NGC 2660, six in NGC 3960, nine in Be 32, seven in NGC 2324, and six in NGC 2477. In NGC 2324 the three stars with $R V$ differing from the cluster average are known long period binaries (Mermilliod et al. 2001) and were retained, while we eliminated the fastrotating star 10360 from further analysis (see Fig. 2). All our targets, except 10360, were observed by Mermilliod et al., and the average difference in $R V$ between our measures and theirs is $+0.03(\mathrm{rms}=0.35) \mathrm{km} \mathrm{s}^{-1}$, computed excluding the binaries. In NGC 2477 we did not examine the two main sequence stars, as done in Paper I in a similar case, because of the lower quality of the spectra and the shallow, broad lines. Stars 36326 and 36385 are indicated as binaries by Eigenbrod et al. (2004) and were retained, while we excluded star 36284 from further analysis on the basis of its differing $R V$, since we had no further information on its possible binarity. The stars in common with Eigenbrod et al. are indicated in Table 2; the average difference in $R V$ between our measures and theirs, excluding the binaries, is -0.02 $(\mathrm{rms}=0.26) \mathrm{km} \mathrm{s}^{-1}$. 


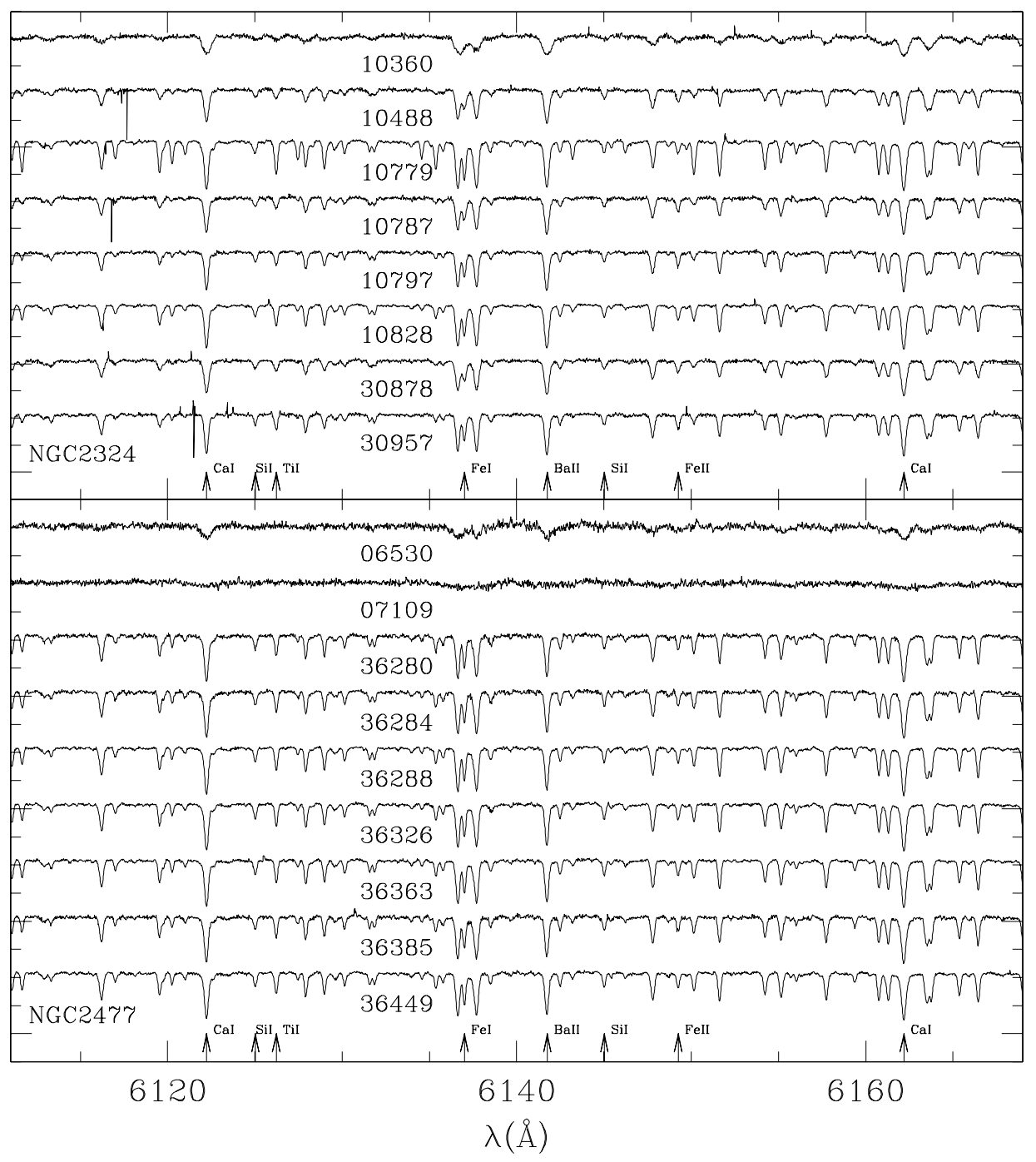

Fig. 2. Example of spectra for NGC 2324 (upper panel) and NGC 2477 (lower panel), shifted to $R V=0 \mathrm{~km} \mathrm{~s}^{-1}$. Notice the wide lines for the giant star 10360 in NGC 2324, indicative of a fast rotation; objects 6530 and 7109 in NGC 2477 are MS stars. A few lines used in our abundance analysis are indicated.

\subsection{Solar analysis and line list}

As described in Paper I, the first important step is the definition of the reference solar abundances, in order to minimize the errors due to uncertainties in the atomic parameters. We adopted the line list by Gratton et al. (2003, hereafter G03), covering the wavelength range $4150-8200 \AA$. Among the elements originally included in that list we study here $\mathrm{Na}, \mathrm{Mg}, \mathrm{Si}, \mathrm{Ca}, \mathrm{Ti}, \mathrm{Cr}$ and Ni. Since Al is lacking in the line list of G03, we added three subordinate Al I doublets used by Carretta et al. (2004a). Barium is not present in the solar line list too, therefore we adopted for this element the solar abundance by Anders \& Grevesse (1989), which is the one adopted inside MOOG.

As already done for NGC 6253 (S07), we determined the abundances of $\mathrm{Fe}$ and (mostly) light elements. Analysis of the $\mathrm{O}$ abundance for the present clusters is deferred to a forthcoming paper, dedicated to the $\mathrm{CNO}$ elements in our entire sample: the $\mathrm{O}$ features usually measurable in giants are the forbidden lines near 6300 and $6363 \AA$, and they require careful and lengthy analysis with synthetic spectra involving also $\mathrm{C}$ and $\mathrm{N}$ abundances (see e.g., Carretta et al. 2005, for the application to a solarmetallicity OC). We have also excluded from the present analysis species like $\mathrm{Mn}, \mathrm{Zn}$ and neutron capture elements, which need an accurate treatment of hyperfine structure (HFS). The only exception is Ba II, since Mashonkina \& Gehren (2001) found that the two subordinate lines of Ba II at 5853 and $6496 \AA$ are free from HFS effects and also from significant departures from LTE.

Sources of oscillator strengths $(\log g f)$ for all the elements investigated (except Al) are reported in Table 8 of G03, where the interested reader can find all details. During the solar analysis we assumed equivalent widths adopted from Rutten \& Van der Zalm (1984), integrating the set with measurements performed by G03 on the solar spectrum atlases by Delbuille et al. (1973) and by Kurucz et al. (1984). The spectral code MOOG has recently been updated in order to allow the user to treat collisional damping with the coefficients from Barklem et al. (2000); see Paper I for details.

We assumed the following effective temperature, surface gravity and microturbulence velocity for the Sun: $T_{\text {eff }}=5779 \mathrm{~K}$, $\log g=4.44$, and $\xi=0.8 \mathrm{~km} \mathrm{~s}^{-1}$ (corresponding to the best-fit in the analysis by Grevesse \& Sauval 1999). Table 3 lists the elements investigated, the number of lines used in the solar analysis (Cols. 1 and 2), and the abundances obtained (Col. 3). The errors are the standard deviations from the average, $\sigma$. Note that $1 \sigma$ clipping was performed during the solar analysis for almost all 
Table 3. Element abundances for the Sun in our analysis, in Anders \& Grevesse (1989) and in Asplund et al. (2005).

\begin{tabular}{lrccc}
\hline \hline El. & $\begin{array}{r}\text { No. } \\
\text { lin. }\end{array}$ & $\begin{array}{c}\log n(\mathrm{X})_{\odot} \\
\text { Our }\end{array}$ & $\begin{array}{c}\log n(\mathrm{X})_{\odot} \\
\text { AG89 }\end{array}$ & $\begin{array}{c}\log n(\mathrm{X})_{\odot} \\
\text { As05 }\end{array}$ \\
\hline $\mathrm{Na}$ I & 7 & $6.29 \pm 0.05$ & 6.33 & $6.17 \pm 0.04$ \\
$\mathrm{Mg}$ I & 4 & $7.62 \pm 0.14$ & 7.58 & $7.53 \pm 0.09$ \\
$\mathrm{Al} \mathrm{I}$ & 3 & $6.33 \pm 0.18$ & 6.47 & $6.37 \pm 0.06$ \\
$\mathrm{Si}$ I & 15 & $7.60 \pm 0.03$ & 7.55 & $7.51 \pm 0.04$ \\
$\mathrm{Ca}$ I & 25 & $6.35 \pm 0.04$ & 6.36 & $6.31 \pm 0.04$ \\
$\mathrm{Ti}$ I & 41 & $4.93 \pm 0.03$ & 4.99 & $4.90 \pm 0.06$ \\
$\mathrm{Ti}$ II & 21 & $5.04 \pm 0.04$ & - & - \\
$\mathrm{Cr}$ I & 61 & $5.66 \pm 0.03$ & 5.67 & $5.64 \pm 0.10$ \\
$\mathrm{Cr}$ II & 21 & $5.78 \pm 0.02$ & - & - \\
$\mathrm{Fe}$ I & 180 & $7.49 \pm 0.04$ & 7.52 & $7.45 \pm 0.05$ \\
$\mathrm{Fe}$ II & 40 & $7.54 \pm 0.03$ & - & - \\
$\mathrm{Ni}$ I & 47 & $6.26 \pm 0.02$ & 6.28 & $6.23 \pm 0.04$ \\
$\mathrm{Ba}$ II & - & - & 2.13 & $2.17 \pm 0.07$ \\
\hline
\end{tabular}

the elements; the only exceptions are $\mathrm{Na}, \mathrm{Mg}$, and $\mathrm{Al}$ for which we used only seven, four and three lines (see also below).

In the last two columns of Table 3 we report for comparison the chemical abundances by Anders \& Grevesse (1989, which are the values adopted inside MOOG), and those of the recent analysis by Asplund et al. (2005) based on 3D-model atmospheres. A general good agreement between our solar abundances and the literature ones can be seen, with the exception of Al. By adopting for this element the same oscillator strengths as in Carretta $(2004 \mathrm{a})$, we find $\log n(\mathrm{Al})=6.33 \pm 0.18$, which is $\sim 0.15$ dex below the "classical" value for the Sun by Anders \& Grevesse (1989), but is in nice agreement with that by Asplund et al. (2005). An Al abundance similar to that by Anders \& Grevesse is obtained for example adopting the $\log g f$ used by Randich et al. (2006), and derived from an inverse solar analysis. In this case we have $\log n(\mathrm{Al})=6.50 \pm 0.03$. As a test, $\mathrm{Al}$ abundances in giant stars were computed assuming either the $\log g f$ by Carretta et al. or those by Randich et al., and we found very similar values of $[\mathrm{Al} / \mathrm{Fe}]$ for all the stars. Therefore, we retain for self-consistency the atomic parameters by Carretta et al. (2004a). The errors on $\log n(\mathrm{Al})$ and $\log n(\mathrm{Mg})$ in the Sun are rather large; this might depend on uncertainties on the $\log g f$ and on the fact that the analysis is based on a very small number of lines. For this reason, the ratio of these elements over Fe in giant stars will be computed from a line-to-line comparison, instead of comparing the average abundance of a star to the solar one (see Sect. 3).

The set of lines used for the analysis of giant stars was also retrieved from G03, and is reported in Table 4: wavelengths, excitation potentials $(E P)$, and $\log g f$ are shown in Cols. $1-3$ and 5-7; several spectral features are in common with those adopted for the Sun, and in these cases the same atomic parameters were assumed. Columns 4 and 8 report the sources for the treatment of damping: computed by MOOG, Barklem et al. (2000), G03, or the classical Unsöld approximation.

We stress that, although the investigation by G03 concerned metal poor halo, thin and thick disk stars, the line list adopted here was accurately tested and employed for solar metallicity open clusters as well (Carretta 2004b, 2005; B06a).

\subsection{Equivalent widths}

Our abundances mainly rely on the spectra centered at $5800 \AA$ (wavelength interval $\sim 4800-6800 \AA$ ), with the exception of $\mathrm{Al}$ and $\mathrm{Na}$ for which we also measured some lines in the spectra centered at $8600 \AA$.
Table 5. Comparison of average $\Delta E W$ values (in $m \AA$ ) for the five clusters.

\begin{tabular}{lcrcr}
\hline \hline Cluster & $\langle\Delta E W\rangle$, rms & $\begin{array}{r}\text { No. } \\
\text { lines }\end{array}$ & $\langle\Delta E W\rangle$, rms & $\begin{array}{r}\text { No. } \\
\text { lines }\end{array}$ \\
& \multicolumn{2}{c}{ only Fe } & & \multicolumn{2}{c}{ other elements } \\
\hline NGC 2660 & $+1.49,4.48$ & 76 & $-2.29,4.98$ & 98 \\
NGC 3960 & $+0.95,2.54$ & 112 & $+1.34,3.61$ & 134 \\
Be 32 & $+1.02,3.75$ & 90 & $+0.26,5.51$ & 106 \\
NGC 2324 & $+2.29,3.46$ & 73 & $+1.35,4.83$ & 95 \\
NGC 2477 & $+0.45,2.67$ & 75 & $+0.78,3.01$ & 103 \\
\hline
\end{tabular}

The continuum tracing and normalization of the spectra were carried out using the task CONTINUUM within IRAF, dividing the spectra in regions of $50 \AA$ and visually checking the output. Equivalent width measurements were carried out with the program SPECTRE, developed by C. Sneden, which performs a Gaussian fitting of the line profiles (see Fitzpatrick \& Sneden 1987). As noted in Paper I this works efficiently also for quite large $E W \mathrm{~s}$, at least up to $150 \mathrm{~m} \AA$, and even more (see Sect. 4.3 and Fig. 5 in Paper I).

As already discussed in Paper I, continuum tracing and $E W$ determination are critical steps for element abundance analysis, in particular for metal rich giant stars, which suffer from heavy line blending.

Random internal errors in $E W \mathrm{~s}$ have been estimated, as in Paper I, by comparing the EWs of two stars with similar parameters. They were computed using stars: 862 and 318 in NGC 2660; c6 and c3 in NGC 3960, 940 and 17 in Be 32, 010797 and 010488 in NGC 2324, 036363 and 036288 in NGC 2477. We considered only the lines in common between the two stars, and rejected significant outliers (see Table 5).

The random errors in $E W$ are represented by $\mathrm{rms} / \sqrt{2}$ (assuming that they can be equally attributed to each of the two stars), therefore their values are $\sim 1.8, \sim 2.7, \sim 3.2, \sim 2.5$, and $\sim 1.9 \mathrm{~mA}$ in the case of Fe, for NGC 3960, Be 32, NGC 2660, NGC 2324, and NGC 2477, respectively. For the other species they are $\sim 2.6, \sim 3.9, \sim 3.5, \sim 3.4$ and $\sim 2.1 \mathrm{~m} \AA$ in the same clusters.

The complete list of measured $E W \mathrm{~s}$ is available only in electronic form through the CDS (Tables 6 to 10, one per cluster, including wavelength, atom, and the $E W$ s for each star).

\subsection{Stellar parameters, metallicity and errors}

Table 11 indicates the final stellar parameters for the stars analyzed in the 5 OCs and the derived metallicities: ID is taken from Sandrelli et al. (1999) for NGC 2660, Prisinzano et al. (2004) for NGC 3960, D'Orazi et al. (2006) for Be 32, and the EIS catalogues for NGC 2324 and NGC 2477; ID 2 is the alternative identification used for the FLAMES pointings, when different. We list here only Fe I, as we optimized gravities to obtain the same value $( \pm 0.01$ dex $)$ for Fe II. For NGC 2660, NGC 3960 and Be 32 we simply report them from Paper I (Table 9) for the reader's convenience.

Input atmospheric parameters for NGC 2324 and NGC 2477 were derived from photometric information in the same way as for the three other clusters, using the values given in Sect. 1. Temperatures and gravities were obtained from spectroscopy. We used the ones based on photometry as initial values, and iterated to obtain excitation and ionization equilibria for iron, which give temperature and gravity. Microturbulent velocities were computed from gravity using the formula in Carretta et al. (2004b). 
Table 11. Stellar parameters and Fe abundances for stars in the three clusters from Paper I and for those in NGC 2324 and NGC 2477.

\begin{tabular}{|c|c|c|c|c|c|c|}
\hline ID & $\begin{array}{l}\text { ID } \\
\text { other }\end{array}$ & $\begin{array}{l}T_{\text {eff }} \\
(\mathrm{K})\end{array}$ & $\log g$ & $\begin{array}{c}\xi \\
\mathrm{km} \mathrm{s}^{-1}\end{array}$ & $\overline{\overline{[\mathrm{Fe} / \mathrm{H}]}}$ & $\sigma$ \\
\hline \multicolumn{7}{|c|}{ NGC 2660} \\
\hline 296 & & 5200 & 3.01 & 1.11 & +0.08 & 0.13 \\
\hline 318 & & 5030 & 2.59 & 1.16 & +0.00 & 0.12 \\
\hline 542 & & 5060 & 2.48 & 1.17 & +0.03 & 0.12 \\
\hline 694 & & 100 & 2.77 & 1.14 & +0.05 & 0.13 \\
\hline 862 & & 5100 & 2.60 & 1.16 & +0.02 & 0.13 \\
\hline \multicolumn{7}{|c|}{ NGC 3960} \\
\hline 310755 & c3 & 4950 & 2.35 & 1.19 & +0.00 & 0.12 \\
\hline 310756 & c4 & 5050 & 2.54 & 1.17 & +0.07 & 0.11 \\
\hline 310757 & $\mathrm{c} 5$ & 4870 & 2.16 & 1.22 & +0.00 & 0.11 \\
\hline 310758 & c6 & 4950 & 2.40 & 1.19 & +0.02 & 0.12 \\
\hline 310760 & $\mathrm{c} 8$ & 5040 & 2.57 & 1.18 & +0.00 & 0.11 \\
\hline 310761 & \multicolumn{5}{|c|}{ Be 32} & 0.12 \\
\hline 533 & 17 & 4830 & 2.22 & 1.21 & -0.31 & 0.12 \\
\hline 997 & 18 & 4850 & 2.27 & 1.21 & -0.27 & 0.13 \\
\hline 787 & 19 & 760 & 2.26 & 1.21 & -0.35 & 0.13 \\
\hline 121 & 25 & 760 & 2.40 & 1.19 & -0.20 & 0.14 \\
\hline 605 & 27 & 4780 & 2.35 & 1.19 & -0.24 & 0.13 \\
\hline 1139 & 45 & 4920 & 3.00 & 1.11 & -0.35 & 0.14 \\
\hline & 938 & 4870 & 2.33 & 1.19 & -0.30 & 0.13 \\
\hline 104 & 940 & 4800 & 2.10 & 1.23 & -0.33 & 0.13 \\
\hline 99 & 941 & 4760 & 2.40 & 1.19 & -0.29 & 0.13 \\
\hline \multicolumn{7}{|c|}{ NGC 2324} \\
\hline 01048 & & 5100 & 2.20 & 1.21 & -0.17 & 0.16 \\
\hline 010779 & & 4300 & 1.00 & 1.37 & -0.14 & 0.17 \\
\hline 010787 & & 5060 & 2.10 & 1.23 & -0.17 & 0.16 \\
\hline 010797 & & 5040 & 2.25 & 1.21 & -0.17 & 0.16 \\
\hline 010828 & & 4750 & 1.65 & 1.29 & -0.10 & 0.16 \\
\hline 030878 & & 5000 & 2.10 & 1.22 & -0.27 & 0.16 \\
\hline 030957 & & 5000 & 1.84 & 1.26 & -0.17 & 0.16 \\
\hline \multicolumn{7}{|c|}{ NGC 2477} \\
\hline 03628 & & 4980 & 2.80 & 1.14 & +0.05 & 0.11 \\
\hline 036288 & & 4970 & 2.68 & 1.15 & +0.05 & 0.11 \\
\hline 036326 & & 5000 & 2.70 & 1.15 & +0.10 & 0.11 \\
\hline 03636 & & 4950 & 2.66 & 1.16 & +0.05 & 0.11 \\
\hline 03638 & & 5030 & 2.67 & 1.15 & +0.07 & 0.11 \\
\hline 036449 & & 4970 & 2.65 & 1.16 & +0.12 & 0.11 \\
\hline
\end{tabular}

The final average metallicities are $[\mathrm{Fe} / \mathrm{H}]=-0.17$ (rms 0.05) dex for NGC 2324 and $[\mathrm{Fe} / \mathrm{H}]=+0.07$ (rms 0.03) dex for NGC 2477, based on 7 and 6 member stars, respectively.

The estimate of the errors in the resulting $\mathrm{Fe}$ abundances has been described in Paper I and in Carretta et al. (2004b). In short, uncertainties on Fe are given by two contributions: $\sigma_{1}$, the standard deviation (rms) from the mean abundance based on the whole set of lines for each star, which represents the effect of random errors in $E W \mathrm{~s}$ and of uncertainties in $\log g f \mathrm{~s}$; and $\sigma_{2}$, resulting from random uncertainties in the stellar parameters $T_{\text {eff }}$, $\log g$ and $\xi$. Random errors in the three parameters were evaluated following the method described by Carretta et al. (2004b); then, we computed typical $\sigma_{2}$ errors in the abundances by varying each parameter at a time (by a quantity corresponding to the random error) and holding the others fixed. The errors $\sigma$ reported in Table 11 (Col. 7) for Fe are the quadratic sum of $\sigma_{1}$ and $\sigma_{2}$.

As for other elements, the uncertainties $\sigma$ on $[\mathrm{X} / \mathrm{Fe}]^{5}$ were computed by quadratically adding the $\mathrm{rms} \sigma_{1}$ found for $[\mathrm{Fe} / \mathrm{H}]$ to the $\sigma_{1}$ found for the $[\mathrm{X} / \mathrm{H}]$ abundance.

\footnotetext{
${ }_{5}$ We use the usual spectroscopic notation: $\log n(\mathrm{~A})$ is the abundance (by number) of the element $\mathrm{A}$ in the scale where $\log n(\mathrm{H})=12 ;[\mathrm{A} / \mathrm{H}]$ is the logarithmic ratio of the abundances of elements $\mathrm{A}$ and $\mathrm{H}$ in the star, minus the same quantity in the Sun.
}

Table 12. Sensitivities of abundances to variations in the atmospheric parameters.

\begin{tabular}{|c|c|c|c|}
\hline & $\begin{array}{c}\sigma_{T \text { eff }} \\
\Delta: \pm 70 \mathrm{~K}\end{array}$ & $\begin{array}{c}\sigma_{\log g} \\
\pm 0.2 \mathrm{dex}\end{array}$ & $\begin{array}{c}\sigma_{\xi} \\
\pm 0.15 \mathrm{~km} \mathrm{~s}^{-1}\end{array}$ \\
\hline \multicolumn{4}{|c|}{ NGC 3960, star c9-310761 } \\
\hline$[\mathrm{Fe} / \mathrm{H}]$ & $+0.06 /-0.04$ & $+0.01 / 0.00$ & $-0.05 /+0.06$ \\
\hline$[\mathrm{Na} / \mathrm{Fe}]$ & $+0.01 /-0.02$ & $-0.05 /+0.04$ & $+0.02 /-0.02$ \\
\hline$[\mathrm{Mg} / \mathrm{Fe}]$ & $-0.01 /+0.01$ & $-0.01 /+0.01$ & $+0.02 /-0.02$ \\
\hline$[\mathrm{Al} / \mathrm{Fe}]$ & $-0.02 /+0.01$ & $-0.02 /+0.02$ & $+0.03 /-0.03$ \\
\hline$[\mathrm{Si} / \mathrm{Fe}]$ & $-0.06 /+0.04$ & $+0.02 /+0.08$ & $+0.01 /-0.03$ \\
\hline$[\mathrm{Ca} / \mathrm{Fe}]$ & $+0.01 /-0.03$ & $-0.05 /+0.03$ & $+0.04 /-0.02$ \\
\hline$[\mathrm{Ti} / \mathrm{Fe}] \mathrm{I}$ & $+0.02 /-0.04$ & $-0.03 /+0.01$ & $-0.02 /+0.01$ \\
\hline$[\mathrm{Ti} / \mathrm{Fe}] \mathrm{II}$ & $-0.07 /+0.06$ & $+0.08 /-0.08$ & $-0.03 /+0.02$ \\
\hline$[\mathrm{Cr} / \mathrm{Fe}] \mathrm{I}$ & $+0.01 /-0.04$ & $-0.03 /+0.00$ & $-0.02 /+0.00$ \\
\hline$[\mathrm{Cr} / \mathrm{Fe}] \mathrm{II}$ & $-0.09 /+0.09$ & $+0.08 /-0.06$ & $+0.00 /-0.01$ \\
\hline$[\mathrm{Ni} / \mathrm{Fe}]$ & $-0.02 /+0.00$ & $+0.01 /-0.02$ & $-0.01 /+0.01$ \\
\hline$[\mathrm{Ba} / \mathrm{Fe}] \mathrm{II}$ & $+0.02 /-0.01$ & $+0.04 /-0.04$ & $-0.10 /+0.08$ \\
\hline \multicolumn{4}{|c|}{ Berkeley 32 , star $17-533$} \\
\hline$[\mathrm{Fe} / \mathrm{H}]$ & $+0.06 /-0.05$ & $+0.01 / 0.00$ & $-0.05 /+0.06$ \\
\hline$[\mathrm{Na} / \mathrm{Fe}]$ & $+0.00 /-0.01$ & $-0.03 /+0.02$ & $+0.02 /-0.02$ \\
\hline$[\mathrm{Mg} / \mathrm{Fe}]$ & $-0.05 /+0.02$ & $-0.05 /-0.02$ & $+0.00 /-0.05$ \\
\hline$[\mathrm{Al} / \mathrm{Fe}]$ & $-0.02 /+0.02$ & $-0.02 /+0.01$ & $+0.04 /-0.04$ \\
\hline$[\mathrm{Si} / \mathrm{Fe}]$ & $-0.06 /+0.06$ & $+0.02 /-0.03$ & $+0.03 /-0.02$ \\
\hline$[\mathrm{Ca} / \mathrm{Fe}]$ & $+0.01 /-0.03$ & $-0.04 /+0.02$ & $-0.01 /+0.01$ \\
\hline$[\mathrm{Ti} / \mathrm{Fe}] \mathrm{II}$ & $-0.08 /+0.06$ & $+0.05 /-0.11$ & $-0.02 /+0.03$ \\
\hline$[\mathrm{Ti} / \mathrm{Fe}] \mathrm{I}$ & $+0.02 /-0.04$ & $-0.02 /+0.02$ & $-0.01 /+0.01$ \\
\hline$[\mathrm{Cr} / \mathrm{Fe}] \mathrm{I}$ & $+0.02 /-0.03$ & $-0.02 /+0.03$ & $-0.01 /+0.01$ \\
\hline$[\mathrm{Cr} / \mathrm{Fe}] \mathrm{II}$ & $-0.10 /+0.08$ & $+0.06 /-0.11$ & $+0.01 /-0.01$ \\
\hline$[\mathrm{Ni} / \mathrm{Fe}]$ & $-0.02 /+0.01$ & $+0.01 /-0.03$ & $+0.00 /+0.00$ \\
\hline$[\mathrm{Ba} / \mathrm{Fe}] \mathrm{II}$ & $+0.02 /-0.02$ & $+0.04 /-0.05$ & $-0.10 /+0.09$ \\
\hline
\end{tabular}

As in Paper I, sensitivities of the derived abundances to variations in the atmospheric parameters were derived for one star per cluster, chosen as representative; they are shown in Table 12. Since they are similar for all OCs, we report in the table only the ones for NGC 3960 and Be 32 as representative of clusters of solar and lower than solar metallicity, respectively.

Finally, we used our spectroscopically derived temperatures, together with the photometry of the observed stars, to derive reddening values. This work is presented in the Appendix.

\section{Abundances}

The results of our abundance measurements for $\mathrm{Na}, \mathrm{Si}, \mathrm{Ca}, \mathrm{Ti}$, $\mathrm{Cr}, \mathrm{Ni}$ and $\mathrm{Ba}$ in the individual stars are presented in Table 13. The results for $\mathrm{Mg}$ and $\mathrm{Al}$ are presented separately in Table 14, where we report the abundances $\log n(\mathrm{X})$ and $[\mathrm{X} / \mathrm{Fe}]$ for the lines in common between the lists adopted for the Sun and for the giants (two for each element), as we did in S07. We performed $1 \sigma$ clipping for all the elements apart from $\mathrm{Na}, \mathrm{Mg}$ and $\mathrm{Al}$, similarly to the Sun, since the line lists include only a small number of features for these elements.

Averages for $\mathrm{Mg}$ and $\mathrm{Al}$ were computed from the $[\mathrm{X} / \mathrm{Fe}]$ values of the various lines for each element, while for the other species we averaged $\log n(\mathrm{X})$ of the various lines in each star and subtracted the mean abundance of the same element obtained for the Sun and the $[\mathrm{Fe} / \mathrm{H}]$ of the star. Cluster mean abundances and their rms are presented in Table 15: as expected, there is no significant star-to-star scatter in the abundances for any element within a given cluster.

The spectral analysis package MOOG works under the assumption of local thermodynamic equilibrium (LTE): this approximation is good for most species, but for $\mathrm{Na}$ it might lead to systematic errors in the determination of abundances. The effects of non-local thermodynamic equilibrium (NLTE) occurring 
Table 15. Average abundances for the five clusters.

\begin{tabular}{|c|c|c|c|c|c|c|c|c|c|c|}
\hline \multirow[t]{2}{*}{ Element } & \multicolumn{2}{|c|}{ NGC 2660} & \multicolumn{2}{|c|}{ NGC 3960} & \multicolumn{2}{|c|}{ Berkeley 32} & \multicolumn{2}{|c|}{ NGC 2324} & \multicolumn{2}{|c|}{ NGC 2477} \\
\hline & mean & $\mathrm{rms}$ & mean & $\mathrm{rms}$ & mean & $\mathrm{rms}$ & mean & $\mathrm{rms}$ & mean & $\mathrm{rms}$ \\
\hline$[\mathrm{Fe} / \mathrm{H}] \mathrm{I}$ & $\overline{0.04}$ & 0.04 & $\overline{0.02}$ & 0.04 & -0.29 & 0.04 & -0.17 & 0.05 & 0.07 & 0.03 \\
\hline$[\mathrm{Na} / \mathrm{Fe}] \mathrm{LTE}$ & 0.13 & 0.04 & 0.16 & 0.02 & 0.14 & 0.08 & 0.36 & 0.06 & 0.17 & 0.02 \\
\hline$[\mathrm{Na} / \mathrm{Fe}]$ NLTE & 0.12 & 0.04 & 0.09 & 0.03 & 0.12 & 0.07 & 0.31 & 0.10 & 0.12 & 0.03 \\
\hline$[\mathrm{Mg} / \mathrm{Fe}] \mathrm{I}$ & 0.15 & 0.04 & 0.13 & 0.05 & 0.27 & 0.08 & 0.25 & 0.13 & 0.07 & 0.13 \\
\hline$[\mathrm{Al} / \mathrm{Fe}] \mathrm{I}$ & -0.08 & 0.10 & -0.06 & 0.06 & 0.11 & 0.10 & 0.00 & 0.08 & -0.01 & 0.04 \\
\hline$[\mathrm{Si} / \mathrm{Fe}] \mathrm{I}$ & 0.00 & 0.03 & 0.04 & 0.05 & 0.12 & 0.04 & 0.06 & 0.11 & 0.05 & 0.03 \\
\hline$[\mathrm{Ca} / \mathrm{Fe}] \mathrm{I}$ & 0.04 & 0.05 & 0.02 & 0.03 & 0.07 & 0.04 & 0.15 & 0.05 & -0.01 & 0.01 \\
\hline$[\mathrm{Ti} / \mathrm{Fe}] \mathrm{I}$ & 0.00 & 0.03 & -0.04 & 0.02 & 0.02 & 0.06 & -0.08 & 0.03 & 0.01 & 0.06 \\
\hline$[\mathrm{Ti} / \mathrm{Fe}] \mathrm{II}$ & 0.06 & 0.06 & 0.06 & 0.04 & 0.15 & 0.04 & 0.08 & 0.09 & 0.05 & 0.07 \\
\hline$[\mathrm{Cr} / \mathrm{Fe}] \mathrm{I}$ & 0.00 & 0.02 & 0.05 & 0.03 & -0.05 & 0.04 & -0.03 & 0.03 & 0.02 & 0.03 \\
\hline$[\mathrm{Cr} / \mathrm{Fe}] \mathrm{II}$ & -0.04 & 0.03 & 0.01 & 0.04 & 0.06 & 0.09 & 0.01 & 0.06 & 0.10 & 0.08 \\
\hline$[\mathrm{Ni} / \mathrm{Fe}] \mathrm{I}$ & -0.03 & 0.02 & -0.01 & 0.03 & 0.00 & 0.04 & -0.09 & 0.02 & 0.00 & 0.04 \\
\hline$[\mathrm{Ba} / \mathrm{Fe}] \mathrm{II}$ & 0.61 & 0.04 & 0.54 & 0.04 & 0.29 & 0.10 & 0.62 & 0.08 & 0.46 & 0.05 \\
\hline$[\alpha / \mathrm{Fe}]$ & 0.01 & 0.02 & 0.01 & 0.04 & 0.07 & 0.05 & 0.04 & 0.12 & 0.02 & 0.03 \\
\hline
\end{tabular}

in stellar atmospheres depend on the evolutionary status, i.e. on surface gravity and temperature, and on opacity, hence on the metallicity. Na abundances were corrected for deviation from LTE following Gratton et al. (1999) and we list in Table 13 both the LTE and NLTE Na abundances.

The average values for $[\alpha / \mathrm{Fe}]$ in the five clusters are given in the last row of Table 15 . They were computed using $\mathrm{Si}, \mathrm{Ca}$, and $\mathrm{Ti}$ that are all explosive $\alpha$-elements coming from type II SNe, and for which we measured a large number of lines. Magnesium was considered less secure, being based on only two lines; however, had we also included this element, our $[\alpha / \mathrm{Fe}]$ values would have been larger only by 0.01 to $0.05 \mathrm{dex}$, still fully consistent with the thin-disk population (see Sect. 3.2). Abundances of $\mathrm{Si}, \mathrm{Ca}$, and $\mathrm{Ti}$ are all about solar, and the same for the three metal-richer clusters, which present a very low rms for $[\alpha / \mathrm{Fe}]$ (see Table 15). These three elements are slightly less stable in the lower metallicity clusters Be 32 and NGC 2324, so that the $[\alpha / \mathrm{Fe}]$ ratios have larger rms. However, also these ratios are similar considering the errors, and nearly solar, as expected for thindisk stars, and in line with other clusters (see next).

Abundances of the two elements of the Fe group, $\mathrm{Cr}$ and $\mathrm{Ni}$, are based on a large set of lines. Both elements show a solar ratio, as expected.

Abundances of $\mathrm{Ba}$ were derived using the three lines indicated in Table 4; besides the two indicated in Sect. 2.1 as free from discernible HFS effects, we measured the $6164.75 \AA$ line; the agreement of this line with the others suggests that HFS effects are of low importance for the kind of stars considered in our analysis.

The only two elements (apart from Fe) for which both neutral and ionized lines are included in the analysis are $\mathrm{Ti}$ and $\mathrm{Cr}$. They are generally in good agreement, an indication of the validity of our atmospheric parameters and/or of the validity of assuming LTE in the analysis. Even in the few cases when the agreement is slightly worse (the lower metallicity clusters Be 32 and NGC 2324), the difference is about 0.15 dex at most ${ }^{6}$. Since our list includes a smaller number of lines for Ti II and Cr II, the results from $\mathrm{Ti}$ I and $\mathrm{Cr}$ I are more reliable and we will use them in the following.

\footnotetext{
${ }^{6}$ As noted by the referee, the generally higher abundance found for ionized species may be an indication of a weak influence of NLTE (see also the discussion in Allende Prieto et al. 2004).
}

\subsection{Comparison with previous works}

Friel et al. (2002), using low resolution spectroscopy, determined metallicities for 4 of the 5 OCs studied here. They found: $[\mathrm{Fe} / \mathrm{H}]=-0.15(\mathrm{NGC} 2324,7$ stars, $\sigma=0.16),[\mathrm{Fe} / \mathrm{H}]=-0.13$ (NGC 2477, 28 stars, $\sigma=0.10),[\mathrm{Fe} / \mathrm{H}]=-0.50(\mathrm{Be} 32,10$ stars, $\sigma=0.04)$, and $[\mathrm{Fe} / \mathrm{H}]=-0.34$ (NGC 3960, 5 stars, $\sigma=0.09$ ). Our metallicities are generally larger by about $0.2 \mathrm{dex}$, but this has been found in other cases: Twarog et al. (1997) determined that the Friel and Janes metallicity scale had an offset with the one defined by DDO photometry, and in particular that it was metal-poorer. This remains seemingly true also with the reassessment done in Friel et al. (2002) where, for instance, they give $[\mathrm{Fe} / \mathrm{H}]=+0.11$ for NGC 6791, a cluster for which all recent literature gives a metallicity well in excess of +0.3 (e.g., Carretta et al. 2007; and Anthony-Twarog et al. 2007, where the origin of this offset is shortly discussed).

For NGC 2324, NGC 2477, NGC 2660, and Be 32 there are no previous study of detailed abundances, while NGC 3960 was recently studied by B06. As already discussed in Paper I, we have three stars in common and the atmospheric parameters we derive are in good agreement. The Fe abundances differ slightly, with our $[\mathrm{Fe} / \mathrm{H}]$ about 0.1 dex larger than that by B06 (on average, see Sect. 6.1 and Table 10 in Paper I) and we find small variations also in the other elements analyzed here.

Figure 3 shows the results of the comparison of the two abundance analyses, both for individual stars (filled triangles, squares and circles) and for the average (open stars) of the three stars. In computing these differences we have taken into account the diverse solar reference values in the two works. As can be seen, differences are always less than 0.1 dex, specially in the averages, with the exception of $\mathrm{Mg}$ (which, however, was treated differently); we regard this as a very good agreement between the two analyses.

\subsection{Are our clusters typical?}

We can compare the clusters analyzed in our survey (note that to the present sample we add NGC 6253, S07) to other open clusters and to the thin-disk population. We chose for this purpose a small selection of open clusters for which recent analysis based on high resolution spectroscopy has been presented by three groups: i) NGC 6819, Cr 261, NGC 6791 (Bragaglia et al. 2001; Carretta et al. 2005, 2007); ii) Be 20, Be 29, Be 31, 


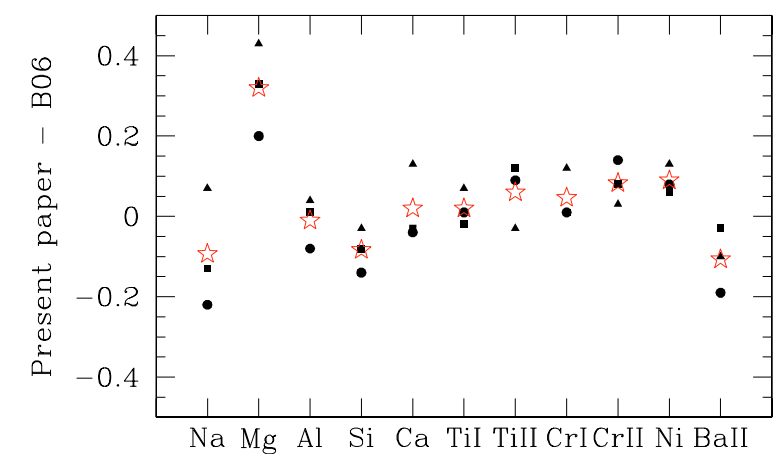

Fig. 3. Comparison for the three NGC 3960 stars in common with B06; small, filled symbols indicate the individual stars (square: c6, triangle: c5, circle: $c 8$ ), while the starred (red) symbols indicate the average for each element.

NGC 2141, M 67 (Yong et al. 2005); iii) Be 22, Be 66 (Villanova et al. 2005) ${ }^{7}$, and Saurer 1 (Carraro et al. 2004) ${ }^{8}$. We decided to limit our comparison sample to these three sets because, together with our OCs, they cover the entire relevant range in cluster properties (e.g., ages from about 0.5 to $9 \mathrm{Gyr}$, Galactocentric distance $R_{\mathrm{GC}}$ from 7 to $21 \mathrm{kpc}$, metallicity from -0.5 to +0.5 ). Furthermore, a higher homogeneity is expected in analyses done in the same paper or by the same authors. As an example of representative abundances for thin-disk field stars, we employed the sample by Soubiran \& Girard (2005), containing several hundreds of stars, reanalyzed and homogenized from several published sources.

Elemental abundances were retrieved from the relevant papers. Age and $R_{\mathrm{GC}}$ for the six clusters in our survey are shown in Table 16, while for the literature OCs they are taken from Bragaglia \& Tosi (2006: Be 29, Cr 261, NGC 6819, Be 22), Friel et al. (2002: NGC 6791, Be 20, Be 31, NGC 2141, M 67), Villanova et al. (2005: Be 66), and Carraro \& Baume (2003: Saurer 1). These values are not completely homogeneous, but the ranking should be right and this is sufficient for our purpose.

Light elements - In all our open clusters $\mathrm{Na}$ is (slightly) enhanced with respect to the solar value; this is a common feature of old OCs (e.g., Friel et al. 2003; Yong et al. 2005), as can be appreciated from Fig. 4a. Our Na abundances are corrected for NLTE as the ones in Bragaglia et al. and Carretta et al.; we found no mention of such a correction in Yong et al., and Soubiran \& Girard have mostly LTE determinations. Since this is beyond the scope of the present paper, we did not try to homogenize the four analyses (for this and the other elements), a tricky work that requires to take into consideration different temperature scales, oscillator strengths, solar reference values etc.

As already noticed by others, there is an apparent discrepancy between clusters and field stars; whether this is due to the different kinds of studied stars (mostly dwarfs in the field sample, mostly giants in the clusters) or employed analyses (model atmospheres, NLTE treatment -e.g., the correction is greater following Mashonkina et al. 2000 rather than Gratton et al. 1999,

\footnotetext{
${ }^{7}$ Given their distance, for these clusters the spectra have rather low $S / N$.

${ }^{8}$ Some of these OCs have been studied also in other papers but we use only the most comprehensive source. For instance, Be 29 was studied also by Carraro et al. (2004) and their results do not generally differ significantly from the Yong et al. (2005) ones, who however studied more elements.
}

Table 16. Summary of properties for the six clusters already examined, in order of decreasing age.

\begin{tabular}{lcrccc}
\hline \hline Cluster & $\begin{array}{c}\text { Age } \\
(\mathrm{Gyr})\end{array}$ & $\begin{array}{r}R_{\mathrm{GC}} \\
(\mathrm{kpc})\end{array}$ & {$[\mathrm{Fe} / \mathrm{H}]$} & $E(B-V)$ & Ref. \\
\hline Be 32 & 5.3 & 10.9 & -0.29 & 0.12 & 1 \\
NGC 6253 & 3.0 & 6.6 & +0.36 & 0.23 & 2 \\
NGC 2477 & 1.0 & 8.9 & +0.07 & 0.30 & 3 \\
NGC 2660 & 0.95 & 8.7 & +0.04 & 0.42 & 2 \\
NGC 3960 & 0.9 & 7.4 & +0.02 & 0.29 & 4 \\
NGC 2324 & 0.6 & 11.7 & -0.17 & 0.19 & 5 \\
\hline
\end{tabular}

etc.) is still unclear. Interestingly, the discrepancy also exists between main sequence and evolved stars in the same cluster: Pasquini et al. (2004) found an enhanced [Na/Fe] ratio for evolved giants with respect to dwarfs in IC 4651, and a similar situation is found for M 67 (e.g. Tautvaisiene et al. 2000 for giants; and Randich et al. 2006, for dwarfs).

The ratio $[\mathrm{Al} / \mathrm{Fe}]$ has been often found enhanced in OCs (e.g., Friel et al. 2005, and references therein). However this is not so clear in our sample: $[\mathrm{Al} / \mathrm{Fe}]$ values go from about -0.1 to +0.1 , without trends with metallicity or age.

$\alpha$-elements - Figure $4 \mathrm{~b}$ indicates that our six clusters and the literature ones follow the run of $[\alpha / \mathrm{Fe}]$ with $[\mathrm{Fe} / \mathrm{H}]$ of thin-disk stars. All $[\alpha / \mathrm{Fe}]$ were computed as average of $\mathrm{Si}, \mathrm{Ca}$ and Ti I, available in all the literature sources considered here (except $\mathrm{Be} 66$, for which the $\mathrm{Si}$ abundance is not given). The situation does not change much if we include also $\mathrm{Mg}$ : $[\alpha / \mathrm{Fe}]$ generally increases, but the variations are of the order of 0.05 dex. We can appreciate from the figure that the lower metallicity OCs studied by Yong et al. (2005), Carraro et al. (2004), and Villanova et al. (2005) seem to have larger $[\alpha / \mathrm{Fe}]$ values; this will be discussed below.

Heavier elements - The Fe-peak elements display a behavior typical of the thin disk; we show Ni in Fig. 4c as an example. Note that the increase in $[\mathrm{Ni} / \mathrm{Fe}]$ in thin-disk stars more metal rich than the Sun could be a feature of the analysis, since it is not seen in the most recent work by Allende Prieto (2007).

Instead, $[\mathrm{Ba} / \mathrm{Fe}]$ seems to show a strong enhancement, especially in NGC 2660 and NGC 3960. We caution that the solar reference value for Ba is taken from Anders \& Grevesse (1989), so the analysis is not strictly differential. Our values appear to be in line with other literature $\mathrm{Ba}$ abundances for OCs that, however, vary a lot: see Fig. 4d, where we used the data from Allende Prieto et al. (2004) for field stars, since Ba was not studied by Soubiran \& Girard (2005).

Radial distributions and age-metallicity considerations - For the open clusters, we show the run of $\alpha$-elements with $R_{\mathrm{GC}}$ in Fig. 5a, and with age in Fig. 5b, while we plot metallicity vs. age in Fig. 5c. There is no apparent dependence of $[\alpha / \mathrm{Fe}]$ on age, or of metallicity on age, while the outer disk clusters seem to display an $\alpha$-enhancement. There is the possibility of an offset between the different samples; however, a similar offset should be present both in the Yong et al. and in the Carraro et al. analyses. In our sample, Be 32 and NGC 2324, the two most external clusters, show a (slightly) larger $[\alpha / \mathrm{Fe}]$ (the effect is increased when also $\mathrm{Mg}$ is considered). An enhanced $[\alpha / \mathrm{Fe}]$ implies a rapid star formation history, where Fe from type Ia SNe has not yet compensated the high $\alpha$ signature of core collapse $\mathrm{SNe}$. This is more typical of the halo evolution, but can also be predicted 

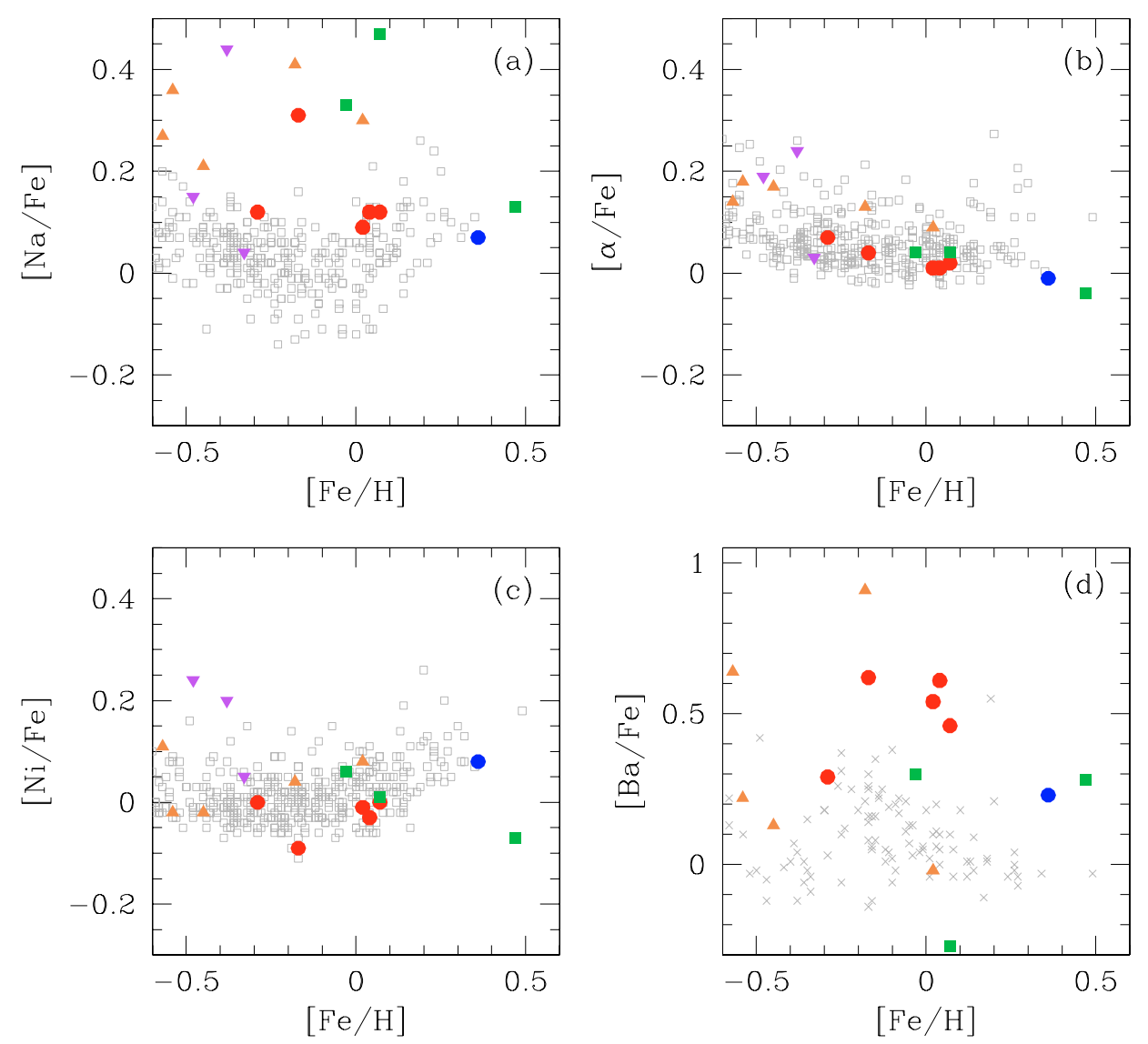

Fig. 4. Comparison of the abundances of $\mathrm{Na}, \mathrm{Ni}$ and $\alpha$ elements for the five clusters in the present paper (red filled circles) and i) thin-disk field stars (grey open squares, Soubiran \& Girard 2005); ii) NGC 6253 (blue filled circle, S07); iii) NGC 6819, Cr 261 and NGC 6791 (green filled squares, Bragaglia et al. 2001; Carretta et al. 2005, 2007); iv) the outer Galactic disk clusters Be 31, Be 29, Be 20, NGC 2141, plus M 67 (orange filled triangles, Yong et al. 2005); the outer Galactic disk clusters Be 22, Be 66 and Saurer 1 (purple inverse triangles, Villanova et al. 2005; Carraro et al. 2004). The comparison of Ba abundances for the same OCs is made with Allende Prieto et al. (2004, grey crosses).

in the outermost regions of the disk by Galactic chemical evolution models, such as model $\mathrm{C}$ by Chiappini et al. (2001). Yong et al. (2005) present a thorough comparison of the OC abundances with those measured in stars of the thin and thick disks, of the halo and the bulge, as well as with chemical evolution model predictions. After discussing the inconsistencies of several scenarios, they suggest a possible merger origin for the formation of the outer disk clusters. This is an interesting subject, worth of further analysis; we will be able to say more on this subject once we will have homogeneously analyzed the two clusters Be 20 and $\mathrm{Be} 29$, the farthest ones in these plots, for which we obtained data with the same FLAMES configuration of the others.

Finally, we show in Fig. $5 \mathrm{~d}$ the run of $[\mathrm{Fe} / \mathrm{H}]$ with $R_{\mathrm{GC}}$ for the OCs already considered. In Paper I and S07 we have shown the radial metallicity distribution based on the low resolution spectroscopy by Friel et al. (2002) and the available high resolution ones (see Paper I for references), and we do not repeat it here. As already noticed by the cited authors, the outer disk OCs display an almost constant metallicity. The OCs presently available in our sample do not add much to that picture. However, NGC 2324 and Be 32 are beyond the about $10 \mathrm{kpc}$ distance from the Galactic centre indicated by Twarog et al. (1997) as separator between inner, solar metallicity clusters and outer, lower metallicity ones. Their metallicities conform to a radial gradient, although they are also compatible with a plateau-like distribution. From the presently existing data it is not clear where the transition from the inner region, where the metallicity clearly shows a radial gradient, and the outer one really is: a larger, finer radial coverage is needed. The three farthest OCs in our sample (Be 20, Be 29, and Tombaugh 2, which is located near the change of slope of the radial metallicity distribution) may be very useful.

\section{Summary}

Using good quality high resolution spectra, we have measured for the first time the atmospheric parameters, the metallicity and the detailed abundances for several elements ( $\mathrm{Na}, \mathrm{Al}, \mathrm{Mg}, \mathrm{Si}$, $\mathrm{Ca}, \mathrm{Ti}, \mathrm{Cr}, \mathrm{Ni}$ and $\mathrm{Ba}$ ) in red clump stars belonging to the OCs NGC 2324 and NGC 2477. Furthermore, we have determined elemental abundances for the stars in the three OCs NGC 2660, NGC 3960, and Berkeley 32 for which the atmospheric parameters and metallicities had been previously presented (Paper I).

These abundances were derived using a list of lines, EPs, $\log g f$ 's and solar reference values that we will adopt also in the following works on the other clusters of our observed sample, in order to produce a homogeneous set of elemental abundances. Table 16 gives a summary of properties for the five clusters here examined (plus NGC 6253); values of [Fe/H] and reddening were derived here (or in Paper I and S07), while ages and Galactocentric radii come from various literature sources 9 .

We do not find indications of star-to-star abundance variations or significant anomalous abundances in any of the five

9 1: Tosi et al. (2007); 2: Bragaglia \& Tosi (2006); 3: Kassis et al. (1997); 4: B06; 5: Kyeong et al. (2001). 

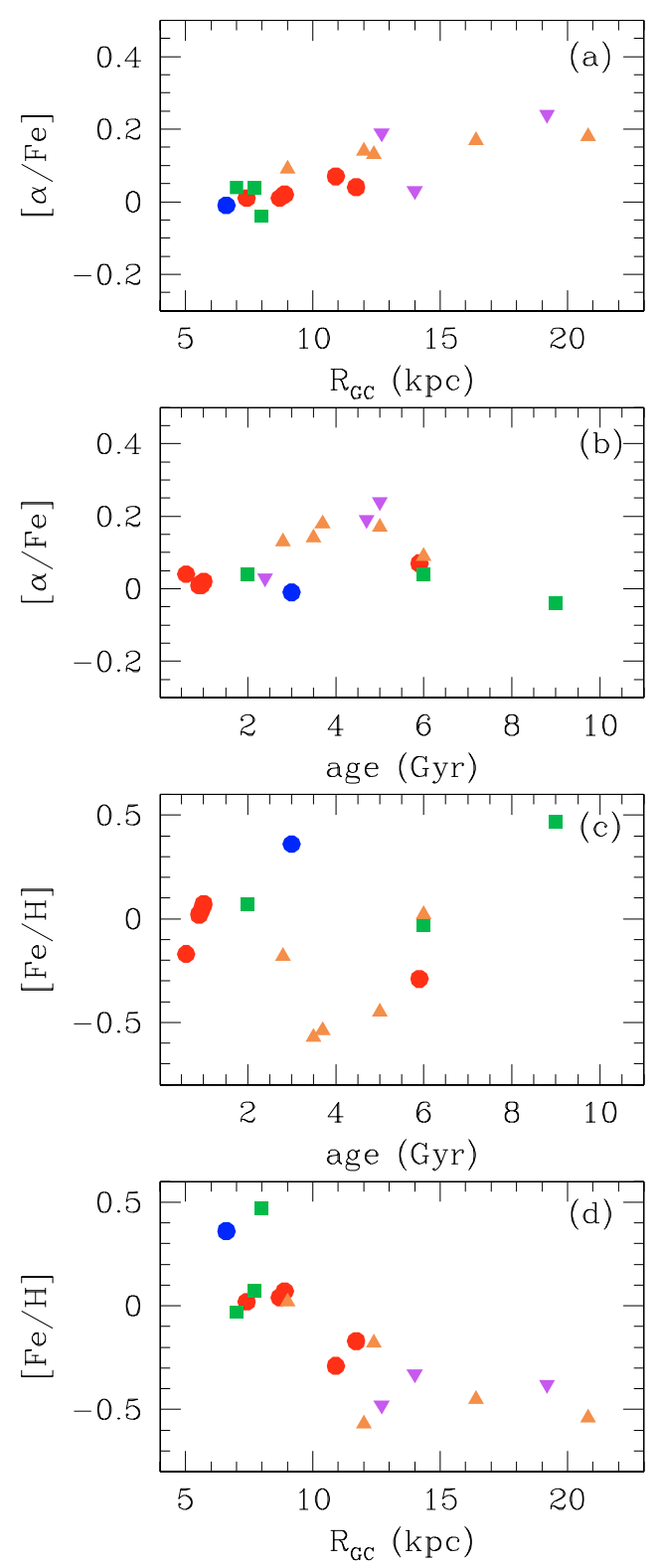

Fig. 5. $\alpha$-elements versus $R_{\mathrm{GC}}($ panel a)) and age (panel b)), metallicity versus age (panel $\mathbf{c}$ )) and $R_{\mathrm{GC}}$ (panel $\mathbf{d}$ )) for the open clusters considered; symbols are as in the preceding figure.

OCs (plus NGC 6253). The elemental abundances for light, $\alpha$ and $\mathrm{Fe}$-peak elements are in line with the run of elemental ratios with $[\mathrm{Fe} / \mathrm{H}]$ of thin-disk field stars. Possible exceptions are $\mathrm{Na}$ and $\mathrm{Ba}$, which also show a large cluster-to-cluster scatter. When we compare the clusters in our sample with others, taken from three homogeneous analyses, we find no evident discrepancies. A more detailed comparison of the values for specific elements with other OCs or disk stars is deferred until we have homogeneously analyzed more clusters in our sample.

Combining our data with the same literature OCs, we do not find any trend of $[\alpha / \mathrm{Fe}]$ with cluster age, while an enhancement for more external, lower metallicity clusters could be present; Be 32 and NGC 2324, the two more external, metal poorer clusters in our sample, could conform to this tendency. A homogeneous analysis of other distant open clusters (e.g., Be 20 and Be 29 for which we already obtained spectra) will allow a more definitive investigation both of this, and of the radial metallicity distribution.
Finally, using the temperatures (derived from the spectroscopic analysis) and a temperature-colour relation we determined individual reddening values for each star (see Appendix). The average for each cluster compares favorably to literature values. Even with a rather small sample of objects, we confirm the presence of differential reddening in NGC 3960 and give some support to its presence in NGC 2477.

Acknowledgements. We thank C. Sneden for useful discussions and the updated release of his code and the referee for costructive comments. This research has made use of the SIMBAD database, operated at CDS, Strasbourg, France, of the NASA's Astrophysics Data System, and of the BDA, created by J. C. Mermilliod and now operated by E. Paunzen at the Institute for Astronomy of the University of Vienna. This publication makes use of data products from the Two Micron All Sky Survey, which is a joint project of the University of Massachusetts and the Infrared Processing and Analysis Center/California Institute of Technology, funded by the National Aeronautics and Space Administration and the National Science Foundation. P.S. acknowledges financial support from the Italian MIUR through PRIN 2003029437, 20040228979 and from the Bologna and Arcetri Observatories.

\section{Appendix A: Reddening}

Our $T_{\text {eff's }}$ are ultimately based on the spectroscopic analysis and come from the Fe excitation equilibrium (see Paper I). We can thus use the photometric data and these temperatures, together with a colour-temperature relation, to determine individual reddening values. We used the Alonso et al. (1999) relations with the metallicities derived in Paper I and adopted $E(V-K)=2.75 E(B-V)$ (Cardelli et al. 1989).

We list in Table A.1 the examined stars. Sources of ID and photometry for NGC 2324 and NGC 2477 are given in Table 2. ID, $B, V$ are taken from Sandrelli et al. (1999) for NGC 2660, Prisinzano et al. (2004) for NGC 3960, D'Orazi et al. (2006) for Be 32; $K$ is from 2MASS (Skrutskie et al. 2006). For each cluster we also indicate the average reddening (and rms), indicating for each star the $T_{\text {eff }}$ and the photometric data and the resulting $E(B-V)$ values, determined from the $(B-V)$ and $(V-K)$ colours. The reddening values derived from the two colours are in good agreement, with the exception of star 296 in NGC 2660, for which the $K$ magnitude is not usable, according to the quality flag.

We clearly see that the reddenings of NGC 2324, NGC 2660, and Be 32 show a scatter smaller - less than half- than that of NGC 3960: this is a confirmation (see Prisinzano et al. 2004, and B06) of the presence of differential reddening in this cluster. We note that for this cluster we derive values of reddening slightly lower than the ones in B06 for the three stars in common. This is partly due to the (minimal) differences in $T_{\text {eff }}$ 's and to the different metallicities, $[\mathrm{Fe} / \mathrm{H}]=-0.12$ in $\mathrm{B} 06$ and +0.02 in Paper I. The largest effect comes from the use of different $B, V$ photometries (see B06 for a comparison of the two). We employ here the values by Prisinzano et al. (2004) that are on average bluer by about 0.05 mag than the corresponding ones in $\mathrm{B} 06$, thus explaining the lower reddening values found here ${ }^{10}$.

For NGC 3960 we have another way to estimate $E(B-V)$, namely using the three main sequence stars observed. They are hot (late A or early F spectral type) and their spectra are not very useful for abundance analysis, since they show very broad and shallow lines, indicative of rotation, and have rather low $\mathrm{S} / \mathrm{N}$. We applied to these stars the method based on the calibration of the interstellar Na I D lines at 5890, $5896 \AA$.

${ }^{10}$ For an unfortunate typographical error, Table 3 in B06 lists the $H$ magnitudes instead of the $K$ ones, but the reddening are correctly computed using $(V-K)$. 
Table A.1. Reddening values derived from $B-V(\mathrm{Col} .7)$ and $V-K(\mathrm{Col} .8)$ and their average (Col. 9).

\begin{tabular}{|c|c|c|c|c|c|c|c|c|}
\hline$\overline{\mathrm{IID}}$ & $\begin{array}{c}\text { Other } \\
\text { ID }\end{array}$ & $\begin{array}{c}T_{\text {eff }} \\
\text { (spectr.) }\end{array}$ & $\overline{\bar{B}}$ & $\overline{\bar{V}}$ & 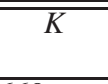 & $\begin{array}{c}E(B-V) \\
(B-V) \\
\end{array}$ & $\begin{array}{c}E(B-V) \\
(V-K) \\
\end{array}$ & $\overline{\langle\langle E(B-V)\rangle}$ \\
\hline \multicolumn{9}{|c|}{ NGC 2660} \\
\hline 296 & & 5200 & 15.798 & 14.552 & 10.636 & 0.427 & - & 0.427 \\
\hline 318 & & 5028 & 15.401 & 14.110 & 10.828 & 0.396 & 0.432 & 0.414 \\
\hline 542 & & 5060 & 15.420 & 14.120 & 10.837 & 0.419 & 0.442 & 0.430 \\
\hline 694 & & 5100 & 15.674 & 14.368 & 11.049 & 0.443 & 0.468 & 0.456 \\
\hline 862 & & 5100 & 15.602 & 14.315 & 11.046 & 0.425 & 0.449 & 0.437 \\
\hline mean & & & & & & 0.421 & 0.448 & 0.433 \\
\hline $\mathrm{rms}$ & & & & & & 0.019 & 0.015 & 0.020 \\
\hline & \multicolumn{8}{|c|}{ NGC 3960} \\
\hline 310755 & c3 & 4950 & 14.799 & 13.514 & 10.357 & 0.354 & 0.362 & 0.358 \\
\hline 310756 & $\mathrm{c} 4$ & 5050 & 14.366 & 13.194 & 10.304 & 0.288 & 0.297 & 0.293 \\
\hline 310757 & $\mathrm{c} 5$ & 4870 & 14.323 & 13.062 & 9.959 & 0.291 & 0.316 & 0.304 \\
\hline 310758 & c6 & 4950 & 14.084 & 12.945 & 10.133 & 0.208 & 0.237 & 0.223 \\
\hline 310760 & c8 & 5040 & 14.224 & 13.060 & 10.162 & 0.275 & 0.297 & 0.286 \\
\hline 310761 & c9 & 5000 & 14.309 & 13.100 & 10.151 & 0.302 & 0.302 & 0.302 \\
\hline mean & & & & & & 0.286 & 0.302 & 0.294 \\
\hline rms & & & & & & 0.047 & 0.040 & 0.043 \\
\hline \multicolumn{9}{|c|}{ Berkeley 32} \\
\hline 533 & 17 & 4830 & 14.754 & 13.667 & 11.028 & 0.140 & 0.138 & 0.139 \\
\hline 997 & 18 & 4850 & 14.730 & 13.661 & 11.069 & 0.133 & 0.128 & 0.131 \\
\hline 787 & 19 & 4760 & 14.800 & 13.709 & 11.024 & 0.107 & 0.129 & 0.118 \\
\hline 121 & 25 & 4760 & 15.387 & 14.242 & 11.450 & 0.161 & 0.168 & 0.165 \\
\hline 605 & 27 & 4780 & 15.511 & 14.372 & 11.607 & 0.166 & 0.166 & 0.166 \\
\hline \multirow[t]{2}{*}{1139} & 45 & 4920 & 16.299 & 15.278 & 12.707 & 0.121 & 0.144 & 0.133 \\
\hline & 938 & 4870 & & 12.501 & 11.027 & & 0.154 & 0.154 \\
\hline 104 & 940 & 4800 & 14.793 & 13.691 & 11.023 & 0.139 & 0.138 & 0.139 \\
\hline 99 & 941 & 4760 & 14.769 & 13.663 & 10.973 & 0.122 & 0.131 & 0.127 \\
\hline mean & & & & & & 0.136 & 0.143 & 0.139 \\
\hline rms & \multirow{2}{*}{\multicolumn{8}{|c|}{ NGC 2324}} \\
\hline & & & & & & & & \\
\hline 010488 & 850 & 5100 & 14.347 & 13.351 & 10.723 & 0.165 & 0.219 & 0.192 \\
\hline 010779 & 2603 & 4300 & - & 12.530 & 9.046 & - & 0.213 & 0.213 \\
\hline 010787 & 2225 & 5060 & 14.444 & 13.422 & 10.803 & 0.172 & 0.204 & 0.188 \\
\hline 010797 & 1006 & 5040 & 14.312 & 13.291 & 10.633 & 0.162 & 0.212 & 0.187 \\
\hline 010828 & 1992 & 4750 & - & 12.508 & 9.571 & - & 0.215 & 0.215 \\
\hline 030878 & 1788 & 5000 & 14.635 & 13.608 & 10.980 & 0.149 & 0.189 & 0.169 \\
\hline 030957 & 2027 & 5000 & 14.374 & 13.325 & 10.670 & 0.171 & 0.199 & 0.185 \\
\hline mean & & & & & & 0.164 & 0.207 & 0.193 \\
\hline \multirow[t]{2}{*}{$\mathrm{rms}$} & \multirow{2}{*}{\multicolumn{8}{|c|}{ NGC 2477}} \\
\hline & & & & & & & & \\
\hline 036280 & 13385 & 4980 & 14.008 & 12.771 & 9.63 & 0.313 & 0.365 & 0.339 \\
\hline 036288 & 4221 & 4970 & 13.428 & 12.231 & 9.36 & 0.268 & 0.264 & 0.266 \\
\hline 036326 & 5035 & 5000 & 13.523 & 12.306 & 9.38 & 0.302 & 0.294 & 0.298 \\
\hline 036363 & 3206 & 4950 & 13.544 & 12.321 & 9.38 & 0.284 & 0.283 & 0.284 \\
\hline 036385 & 2061 & 5030 & 13.914 & 12.710 & 9.75 & 0.303 & 0.316 & 0.310 \\
\hline 036449 & 8039 & 4970 & 13.546 & 12.320 & 9.34 & 0.297 & 0.304 & 0.301 \\
\hline mean & & & & & & 0.295 & 0.304 & 0.299 \\
\hline rms & & & & & & 0.016 & 0.035 & 0.025 \\
\hline
\end{tabular}

Munari \& Zwitter (1997) calibrated the relation between the $E W$ s of these lines and reddening, and we applied their Table 2 to the $E W \mathrm{~s}$ of the Na I D1 lines in the three MS stars, measured using the task SPLOT in IRAF. In particular, since the interstellar absorption shows several components (see Fig. A.1), with the three most prominent ones having radial velocities of about -3 , +26 and $+40 \mathrm{~km} \mathrm{~s}^{-1}$, we determined the reddening contributed by each component and then summed. We obtained the following values for $E(B-V)$ : 0.261 (star 300672, or ms2), 0.189 (301103, or ms6), and 0.203 (301142, or ms7). Their mean is $E(B-V)=0.218, \mathrm{rms} 0.038$. These values are lower than found above for the other stars, but this appears a systematic effect due to the very different methods and possibly to the non ideal spectral type of our stars (Munari \& Zwitter 1997, used O and early B stars). However, these results point to a differential reddening too.

In the case of NGC 2477, for which differential reddening had been advocated by Hartwick et al. (1972), Kassis et al. (1997), and Eigenberg et al. (2004), we do not find any strong confirming evidence. However, our measure is based only on a few stars and the dispersion around the mean value is slightly larger than in the three other clusters. The extent of differential reddening is an open question, that could be addressed by further spectroscopic observations or by synthetic colour-magnitude diagrams trying to reproduce the width of the observed sequences (e.g., Bragaglia \& Tosi 2006). 


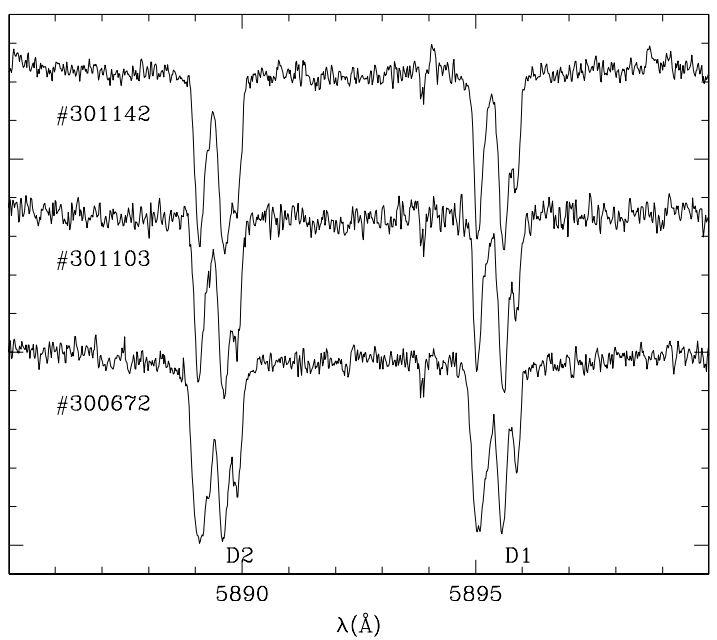

Fig. A.1. Interstellar Na I D1 and D2 lines for the three MS stars in NGC 3960: multiple components are visible, and the dominant ones are found at $R V \mathrm{~s}$ of about $-3,26$ and $40 \mathrm{~km} \mathrm{~s}^{-1}$.

Table A.2. Reddening values for the five clusters compared to recent literature papers.

\begin{tabular}{lcl}
\hline \hline Cluster & $\begin{array}{c}E(B-V) \\
(\mathrm{rms})\end{array}$ & Literature \\
\hline NGC 2660 & 0.433 & $0.40 \pm 0.02(\mathrm{~S}, Z=0.02)$ \\
& $(0.020)$ & $0.37 \pm 0.02(\mathrm{Tw},[\mathrm{Fe} / \mathrm{H}]=-0.18)$ \\
NGC 3960 & 0.294 & $0.29(\mathrm{P}, Z=0.01$, diff. $)$ \\
& $(0.043)$ & $0.29 \pm 0.02(\mathrm{~B}, Z=0.01-0.02$, diff. $)$ \\
& & $0.33(\mathrm{M})$ \\
& & $0.30 \pm 0.01(\mathrm{Tw}, \mathrm{Fe} / \mathrm{H}]=-0.17)$ \\
Berkeley 32 & 0.139 & $0.10(\mathrm{D}, Z=0.008)$ \\
& $(0.017)$ & $0.10(\mathrm{H}, Z=0.008)$ \\
& & $0.16(\mathrm{KM},[\mathrm{Fe} / \mathrm{H}]=-0.4)$ \\
& & $0.08(\mathrm{R},[\mathrm{Fe} / \mathrm{H}]=-0.2)$ \\
NGC 2324 & 0.193 & $0.12(\mathrm{~T}, Z=0.008)$ \\
& $(0.016)$ & $0.25(\mathrm{Ki},[\mathrm{Fe} / \mathrm{H}]=-0.3)$ \\
NGC 2477 $]=-0.31)$ & $0.2-0.4(\mathrm{~K},[\mathrm{Fe} / \mathrm{H}]=-0.05)$ \\
& 0.299 & \\
\hline
\end{tabular}

Finally, we show in Table A.2 the comparison of our findings with literature data for the five clusters $^{11}$; for each reference paper we also indicate the assumed metallicity, if available. The comparison looks very favourable, a further indication that our $T_{\text {eff's are well determined. }}$

We did not compare these reddening values to the ones based on the IR maps by Schlegel et al. (1998), since the latter are not reliable for objects near the Galactic plane, as cautioned by the authors. Furthermore, these OCs are quite close, and they may intercept only part of the absorption in their directions (see e.g., B06, Sect. 3, for an explanation of the difference between the Schlegel et al.'s values and the ones consistently found by every other analysis of NGC 3960).

\section{References}

Allende Prieto, C. 2007, in The Metal-rich Universe, ed. G. Israelian, \& A. Herrero, in press [arXiv: astro-ph/0612200]

Allende Prieto, C., Barklem, P. S., Lambert, D. L., \& Cunha, K. 2004, A\&A, 420, 183

11 B: Bragaglia et al. (2006); D: D’Orazi et al. (2006); H: Hasegawa et al. (2004); K: Kassis et al. (1997); KM: Kaluzny \& Mazur (1991); Ky: Kyeong et al. (2001); M: Mermilliod et al. (2001); P: Prisinzano et al. (2004); Pi: Piatti et al. (2004); R: Richtler \& Sagar (2001); S: Sandrelli et al. (1999); T: Tosi et al. (2007); Tw: Twarog et al. (1997).
Alonso, A., Arribas, S., \& Martínez-Roger, C. 1999, A\&AS, 140, 261 Anders, E., \& Grevesse, N. 1989, GeCoA, 53, 197

Anthony-Twarog, B. J, Twarog, B. A., \& Mayer, L. 2007, AJ, 133, 1585

Asplund, M., Grevesse, N., \& Sauval, A. J. 2005, in Cosmic Abundances as Records of Stellar Evolution and Nucleosynthesis, ed. T. G. Barnes, \& F. N. Bash, ASP Conf. Ser., 336, 25

Barklem, P. S., Piskunov, N., \& O’Mara, B. J. 2000, A\&A, 363, 1091

Bertelli, G., Bressan, A., Chiosi, C., Fagotto, F., \& Nasi, E. 1994, A\&A, 106, 275

Bragaglia, A., \& Tosi, M. 2006, AJ, 131, 1544

Bragaglia, A., Tosi, M., Carretta, E., et al. 2006, 366, 1493 (B06)

Cardelli, J. A., Clayton, G. C., \& Mathis, J. S. 1989, ApJ, 345, 245

Carraro, G., \& Baume, G. 2003, MNRAS, 346, 18

Carraro, G., Bresolin, F., Villanova, S., et al. 2004, AJ, 128, 1676

Carretta, E., Gratton, R. G., Bragaglia, A., Bonifacio, P., \& Pasquini, L. 2004a, A\&A, 416, 925

Carretta, E., Bragaglia, A., Gratton, R. G., \& Tosi, M. 2004b, A\&A, 422, 951

Carretta, E., Bragaglia, A., Gratton, R. G., \& Tosi, M. 2005, A\&A, 441, 131

Carretta, E., Bragaglia, A., \& Gratton, R. G. 2007, A\&A, 437, 129

Chiappini, C., Matteucci, F., \& Romano, D. 2001, ApJ, 554, 1044

Delbouille, L., Roland, G., \& Neven, L. 1973, Atlas photometrique du spectre solaire de $\lambda 3000$ a $\lambda 10000^{\prime \prime}$, Université de Liege, Institut d'Astrophysique

D’Orazi, V., Bragaglia, A., Tosi, M., Di Fabrizio, L., \& Held, E. V. 2006, MNRAS, 368, 471

Eigenbrod, A., Mermilliod, J.-C., Clariá, J. J., Andersen, J., \& Mayor, M. 2004, A\&A, 423, 189

Fitzpatrick, M. J., \& Sneden, C. 1987, BAAS, 19, 1129

Freeman, K., \& Bland-Hawthorn, J. 2002, ARA\&A, 40, 487

Friel, E. D. 1995, ARA\&A, 33, 381

Friel, E. D. 2006, in Chemical abundances and mixing in the Milky Way and its satellites, ESO Astrophysics Symposia, ed. S. Randich, \& L. Pasquini, 3

Friel, E. D., \& Janes, K. A. 1983, A\&A, 267, 75

Friel, E. D., Janes, K. A., Tavarez, M., et al. 2002, AJ, 124, 2693

Friel, E. D., Jacobson, H. R., Barrett, E., et al. 2003, AJ, 126, 2372

Gratton, R. G., Carretta, E., Eriksson, L., \& Gustaffson, B. 1999, A\&A, 350, 955

Gratton, R. G., Carretta, E., Claudi, R., Lucatello, S., \& Barbieri, M. 2003, A\&A, 404, 187 (G03)

Grevesse, N., \& Sauval, A. J. 1999, A\&A, 347, 348

Hartwick, F. D. A., Hesser, J. E., \& McClure, R. D. 1972, AJ, 113, 1723

Hasegawa, T., Malasan, H. L., Kawakita, H., et al. 2004, PASJ, 56, 295

Janes, K. A., \& Phelps, R. L. 1994, AJ, 108, 1773

Kaluzny, J., \& Mazur, B. 1991, AcA, 41, 167

Kassis, M., Janes, K. A., Friel, E. D., \& Phelps, R. L. 1997, AJ, 113, 1723

Kyeong, J.-M., Byun, Y.-I., \& Sung, E. C. 2001, JKAS, 34, 143

Kurucz, R. L. 1993, CD-ROM, No. 9

Kurucz, R. L., Furenlid, I., Brault, J., \& Testerman, L. 1984, Solar Flux Atlas from 296 to $1300 \mathrm{~nm}$, NOAO Atlas, No. 1

Mashonkina, L., \& Gehren, T. 2001, A\&A, 376, 232

Mashonkina, L. I., Shimanskii, V. V., \& Sakhibullin, N. A. 2000, Astron. Rep., 44, 790

Mermilliod, J.-C., Clariá, J. J., Andersen, J., Piatti, A. E., \& Mayor, M. 2001, A\&A, 375, 30

Momany, Y., Vandame, B., Zaggia, S., et al. 2001, A\&A, 379, 436

Munari, U., \& Zwitter, T. 1997, A\&A, 318, 269

Panagia, N., \& Tosi, M. 1981, A\&A, 96, 306

Pasquini, L., Avila, G., Blecha, A., et al. 2002, The Messenger, 110, 1

Pasquini, L., Randich, S., Zoccali, M., et al. 2004, A\&A, 424, 951

Piatti, A. E., Clariá, J. J., \& Ahumada, A. V. 2004, A\&A, 421, 991

Prisinzano, L., Micela, G., Sciortino, S., \& Favata, F. 2004, A\&A, 417, 945

Randich, S., Bragaglia, A., Pastori, L., et al. 2005, The Messenger, 121, 18

Randich, S., Sestito, P., Primas, F., Pasquini, L., \& Pallavicini, R. 2006, A\&A, 450,557

Richtler, T., \& Sagar, R. 2001, BASI, 29, 53

Rutten, R. J., \& Van der Zalm, E. B. J. 1984, A\&AS, 55, 171

Sandrelli, S., Bragaglia, A., Tosi, M., \& Marconi, G. 1999, MNRAS, 309, 739

Schlegel, D. J., Finkbeiner, D. P., \& Davis, M. 1998, ApJ, 500, 525

Sestito, P., Bragaglia, A., Randich, S., et al. 2006, A\&A, 458, 121 (Paper I)

Sestito, P., Randich, S., \& Bragaglia, A. 2007, A\&A, 465, 185 (S07)

Skrutskie, M. F., et al. 2006, AJ, 131, 1163

Sneden, C. A. 1973, ApJ, 184, 839

Soubiran, C., \& Girard, P. 2005, A\&A, 139, 151

Tautvaisiene, G., Edvardsson, B., Tuominen, I., \& Ilyin, I. 2000, A\&A, 360, 499

Tosi, M., Bragaglia, A., \& Cignoni, M. 2007, MNRAS, 378, 730

Twarog, B. A., Ashman, K. M., \& Anthony-Twarog, B. J. 1997, AJ, 114, 2556

Unsöld, A. 1955, Physik der Sternatmosphären (Berlin: Springer-Verlag)

Villanova, S., Carraro, G., Bresolin, F., \& Patat, F. 2005, AJ, 130, 652

Yong, D., Carney, B. W., \& Teixera de de Almeida, M. L. 2005, AJ, 130, 597 